\title{
A Review on the Use of Artificial Intelligence in Spinal Diseases
}

\author{
Parisa Azimi ${ }^{1}$, Taravat Yazdanian ${ }^{2}$, Edward C. Benzel $^{3}$, Hossein Nayeb Aghaei ${ }^{1}$, \\ Shirzad Azhari ${ }^{1}$, Sohrab Sadeghi ${ }^{1}$, Ali Montazeri ${ }^{4}$ \\ ${ }^{1}$ Department of Neurosurgery, Shahid Beheshti University of Medical Sciences, Tehran, Iran \\ ${ }^{2}$ School of Medicine, Capital Medical University, Beijing, China \\ ${ }^{3}$ Department of Neurosurgery, Cleveland Clinic Foundation, Cleveland, OH, USA \\ ${ }^{4}$ Mental Health Research Group, Health Metrics Research Centre, Iranian Institute for Health Sciences Research, ACECR, Tehran, Iran
}

\begin{abstract}
Artificial neural networks (ANNs) have been used in a wide variety of real-world applications and it emerges as a promising field across various branches of medicine. This review aims to identify the role of ANNs in spinal diseases. Literature were searched from electronic databases of Scopus and Medline from 1993 to 2020 with English publications reported on the application of ANNs in spinal diseases. The search strategy was set as the combinations of the following keywords: "artificial neural networks," "spine," "back pain," "prognosis," "grading," "classification," "prediction," "segmentation," "biomechanics," "deep learning," and "imaging." The main findings of the included studies were summarized, with an emphasis on the recent advances in spinal diseases and its application in the diagnostic and prognostic procedures. According to the search strategy, a set of 3,653 articles were retrieved from Medline and Scopus databases. After careful evaluation of the abstracts, the full texts of 89 eligible papers were further examined, of which 79 articles satisfied the inclusion criteria of this review. Our review indicates several applications of ANNs in the management of spinal diseases including (1) diagnosis and assessment of spinal disease progression in the patients with low back pain, perioperative complications, and readmission rate following spine surgery; (2) enhancement of the clinically relevant information extracted from radiographic images to predict Pfirrmann grades, Modic changes, and spinal stenosis grades on magnetic resonance images automatically; (3) prediction of outcomes in lumbar spinal stenosis, lumbar disc herniation and patient-reported outcomes in lumbar fusion surgery, and preoperative planning and intraoperative assistance; and (4) its application in the biomechanical assessment of spinal diseases. The evidence suggests that ANNs can be successfully used for optimizing the diagnosis, prognosis and outcome prediction in spinal diseases. Therefore, incorporation of ANNs into spine clinical practice may improve clinical decision making.
\end{abstract}

Keywords: Spine; Review; Artificial neural networks

\section{Introduction}

Artificial neural network (ANN) models represent a mathematical rendition of the human nervous system that have been broadly applied to solve various nonlinear problems in the biomedical arena $[1,2]$. ANN is a machine-learning technique adept at learning the relationships between specified input and output variables.

Received Apr 3, 2020; Revised Apr 10, 2020; Accepted Apr 12, 2020

Corresponding author: Parisa Azimi

Neuroscience Research Center, Shahid Beheshti University of Medical Sciences, Arabi Ave, Daneshjoo Blvd, Velenjak, Tehran 1983963113, Iran

Tel: +98-21-2243-9770, Fax: +98-21-2243-3455, E-mail: parisa.azimi@gmail.com 
Neural networks have been used predominantly for pattern-recognition regarding prediction and classification. The history and theory of ANNs has been reported in detail elsewhere [1-4]. In addition, the advantages and disadvantages of ANN have also been previously reported by us $[1,2]$. ANN is a promising field with numerous applications across various branches of medicine wherein it serves as a decision support tool to provide economic solutions to time and resource management [5]. Recently, artificial intelligence and related algorithms have facilitated rapid advances in the assessment of spinal diseases [2,5]. Moreover, ANNs are applied for clinical diagnosis, prognosis, outcome prediction following spinal surgery, research, and biomechanical assessments of spinal diseases [2]. However, there has been little utilization of ANNs in spine clinical practice. Given the recent advances in the management of spinal diseases and the fundamental role of decision, this comprehensive review is conducted aiming to describe the ANN-aided decision support system for management of spinal diseases, including diagnosis, prognosis, and outcome prediction.

\section{Methods}

ANN based methodology has been reported in detail elsewhere $[1,2]$.

\section{Search strategy}

A detailed search of original articles was performed on Medline (through the PubMed search engine) and Scopus (Elsevier) databases to identify the applications of ANNs diagnosis, prediction, and prognosis of spinal disease. The review is intended to include all the full-text publications in the English biomedical journals. The following combinations of keywords were searched within the titles and abstracts: "artificial neural networks," "spine," "back pain," "prognosis," "grading," "classification," "prediction," "segmentation," "biomechanics," "deep learning," and "imaging." The structural keywords were selected due to their likelihood of being mentioned in either the title or the abstract of relevant articles. Since the first study of ANNs in spine diseases published in 1993, we performed a comprehensive search covering the period 1993 to 2020. An initial search was carried out in November 2019 and updated thrice in 2020 (January, February, and March).

\section{Inclusion and exclusion criteria}

All research articles on ANNs in spinal diseases were screened in the Scopus and Medline databases. Each article was independently reviewed by two reviewers and disagreements were sent to each other for resolution, only the articles emphasize on the most recent advances and their application in the spinal diseases were included. Publications on other disease conditions or animal studies were excluded.

\section{Data synthesis}

The findings from the all identified studies were summarized in a descriptive table, including authors' names, publication year, study setting, study sample, disease conditions (if relevant data is available), and main results or conclusions. Subsequently, the findings were sorted chronologically.

\section{Results}

\section{Statistics}

The reviewers identified and screened 3,653 unique abstracts. After screening, 3,564 papers were found to be irrelevant. Then, the remaining 89 papers were examined and the full text were reviewed for eligibility criteria. Ultimately, we included 79 studies on qualitative analysis. The flowchart of the literature review process is illustrated in Fig. 1. Overall, we pursued four categories of studies, namely diagnosis, progression, outcome prediction, and use of ANNs in the biomechanical assessments of spinal diseases. The main findings were grouped and presented as follows [6-81].

\section{Diagnosis}

In spinal diseases, ANNs have been successfully tested for diagnosis of pediatric low back pain $[6,9,11]$, normal and abnormal cervical spine vertebra [8], scoliosis spinal deformity $[7,10]$, and identification of risk factors associated with the development of complications following posterior lumbar spine fusion [23]. Besides, artificial intelligence models have been employed for medical image analysis assessment, such as those portrayed in the Table 1. 


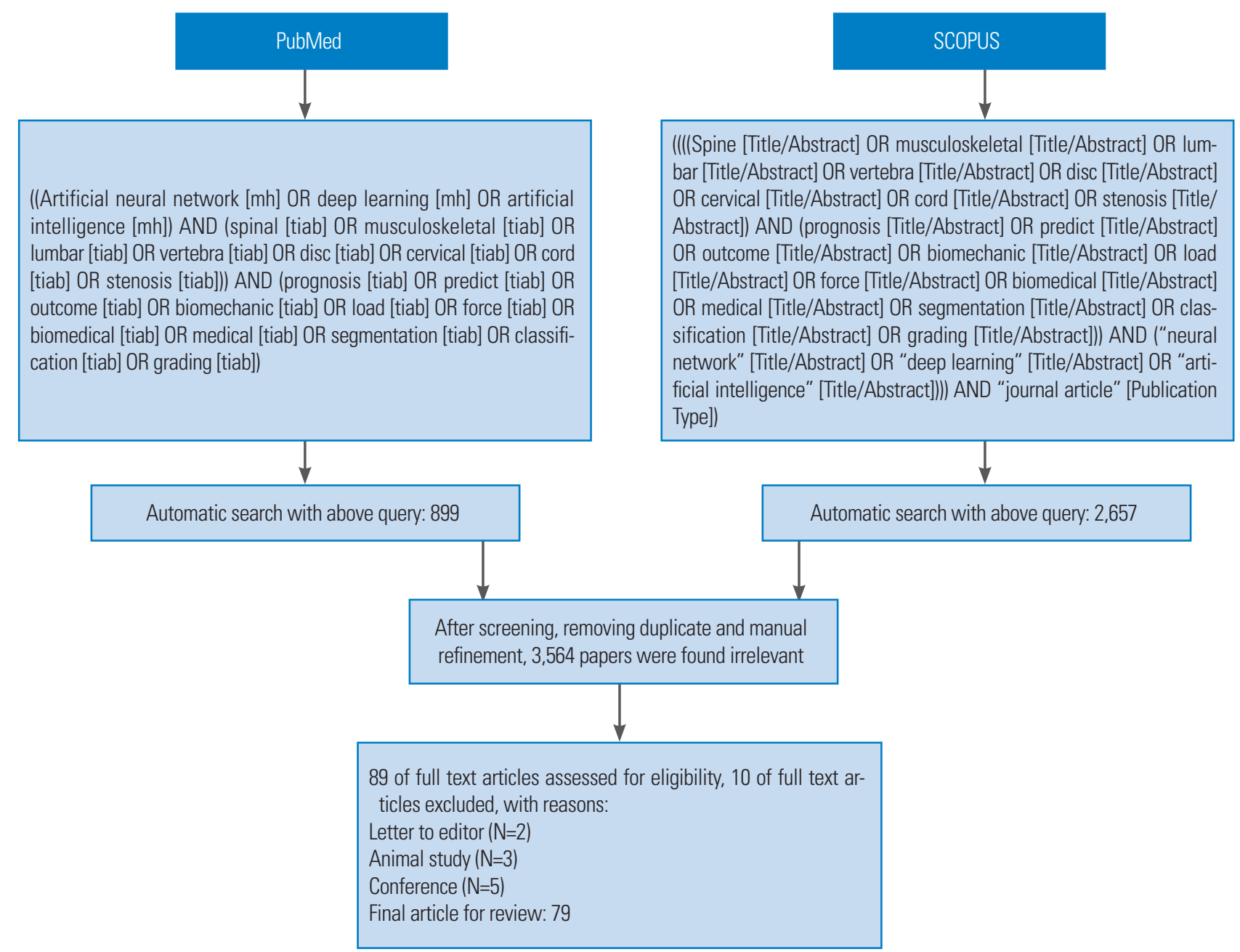

Fig. 1. Flowchart of literature search, selection, and identification.

\section{Spinal prognosis}

In addition to the application of in identifying the patients with high risk of hypotension during spinal anesthesia [52], ANNS have been tested to determine the prognosis of low back pain [51,53] aiming to automatically predict (and identify risk factors for) the complications following posterior lumbar spine fusion surgery [16], and to develop and evaluate a set of predictive models for common adverse events after spine surgery [57]. Also, ANNs are useful for developing novel computational tools to predict clinical outcomes, return to work, physical disability, occurrence of complications, readmission rates, walking ability, discharge, and disposition following spine surgery $[54,55,58]$. Neural network techniques have also been applied to develop predictive algorithms for postoperative complications following anterior cervical discectomy and fusion [56], and to evaluate clinically relevant improvement in leg pain, back pain and functional disability after lumbar disc herniation (LDH) surgery [59], and to automatically quantify muscle fat infiltration following whiplash injury [62]. In addition, ANNs have been shown to accurately predict survival, discharge and hospital readmission rates following spinal metastasis surgery $[57,60,61]$, to predict discharge to rehabilitation and unplanned readmissions in patients receiving spinal fusion [63], and to predict prolonged opioid prescription after surgery for LDH [64]. Last but not least, ANNs have been used to predict the survival rate following a spinopelvic chondrosarcoma diagnosis [65] and to predict the occurrence of four types of major complications, namely cardiac complications, wound complications, venous thromboembolism, and mortality in the patients undergoing spine fusion, and it has achieved better results than 


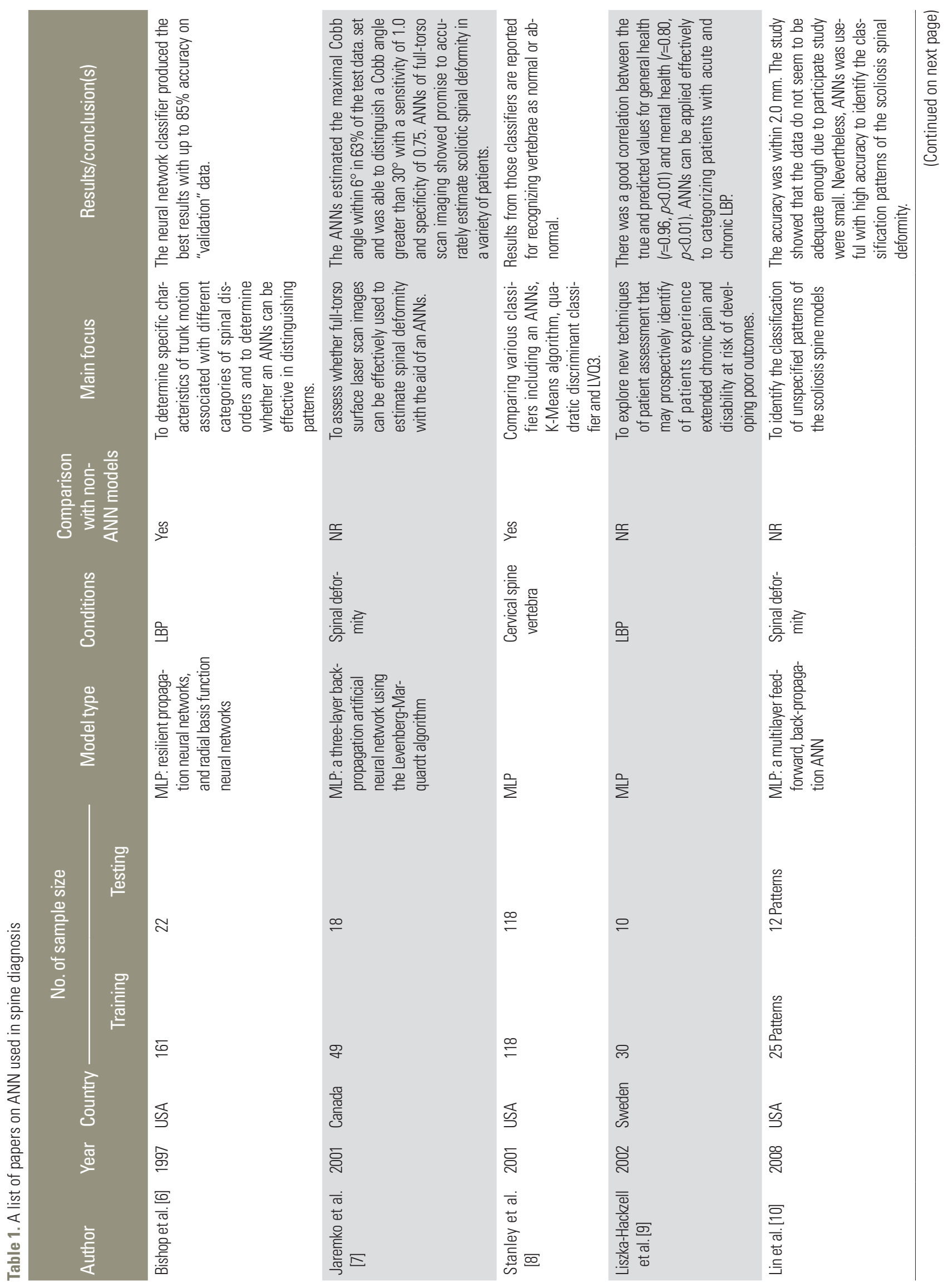




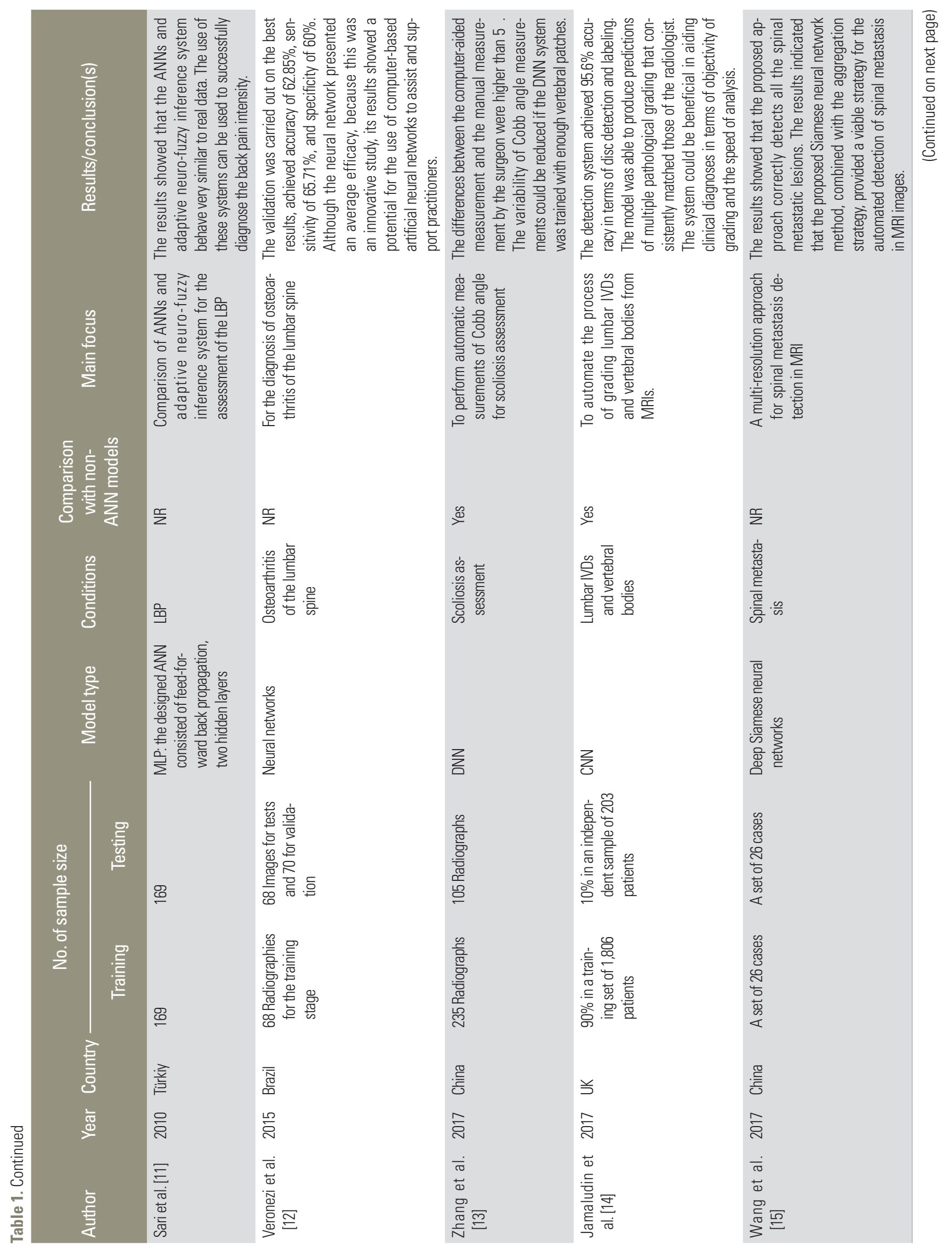




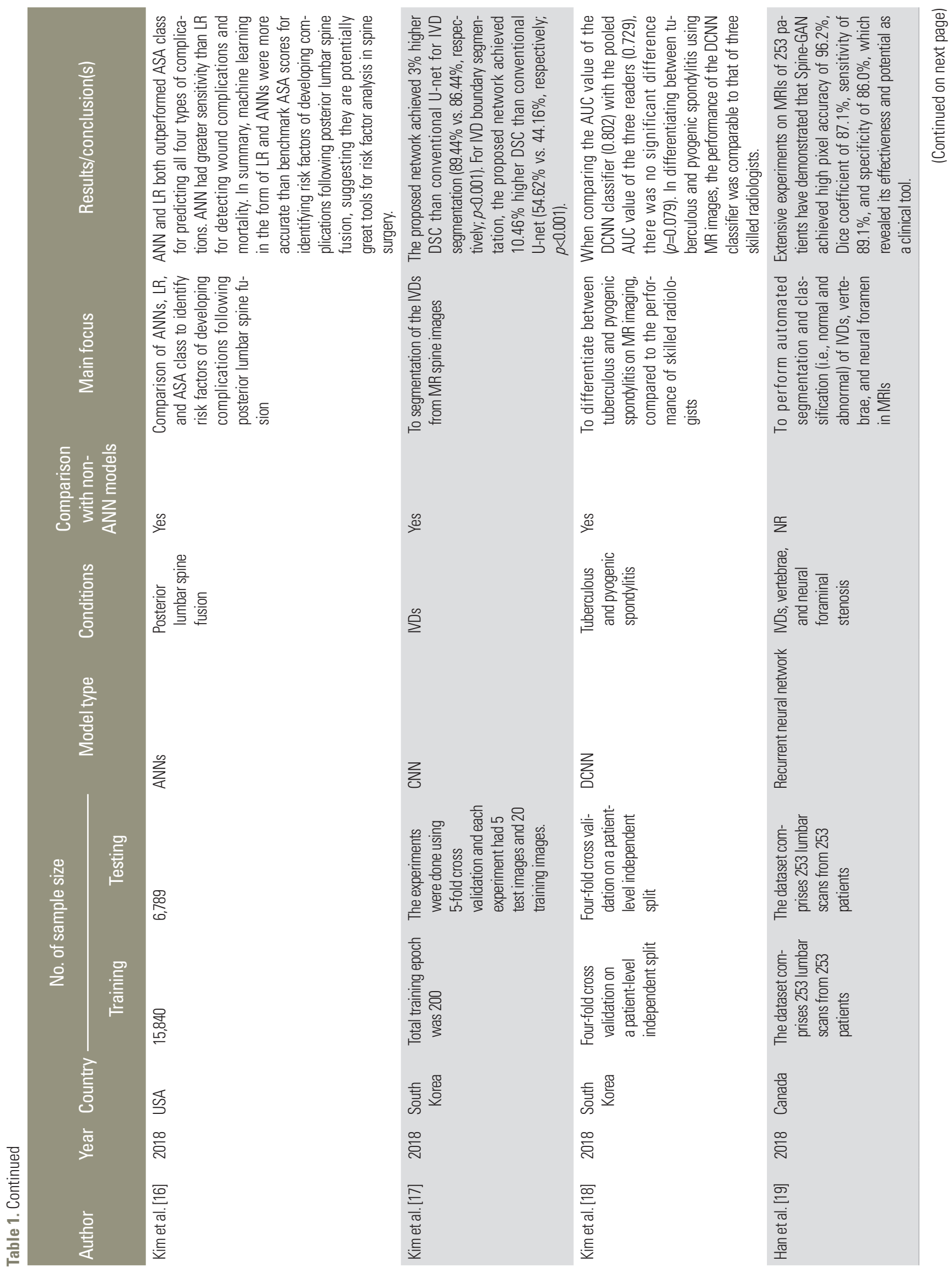




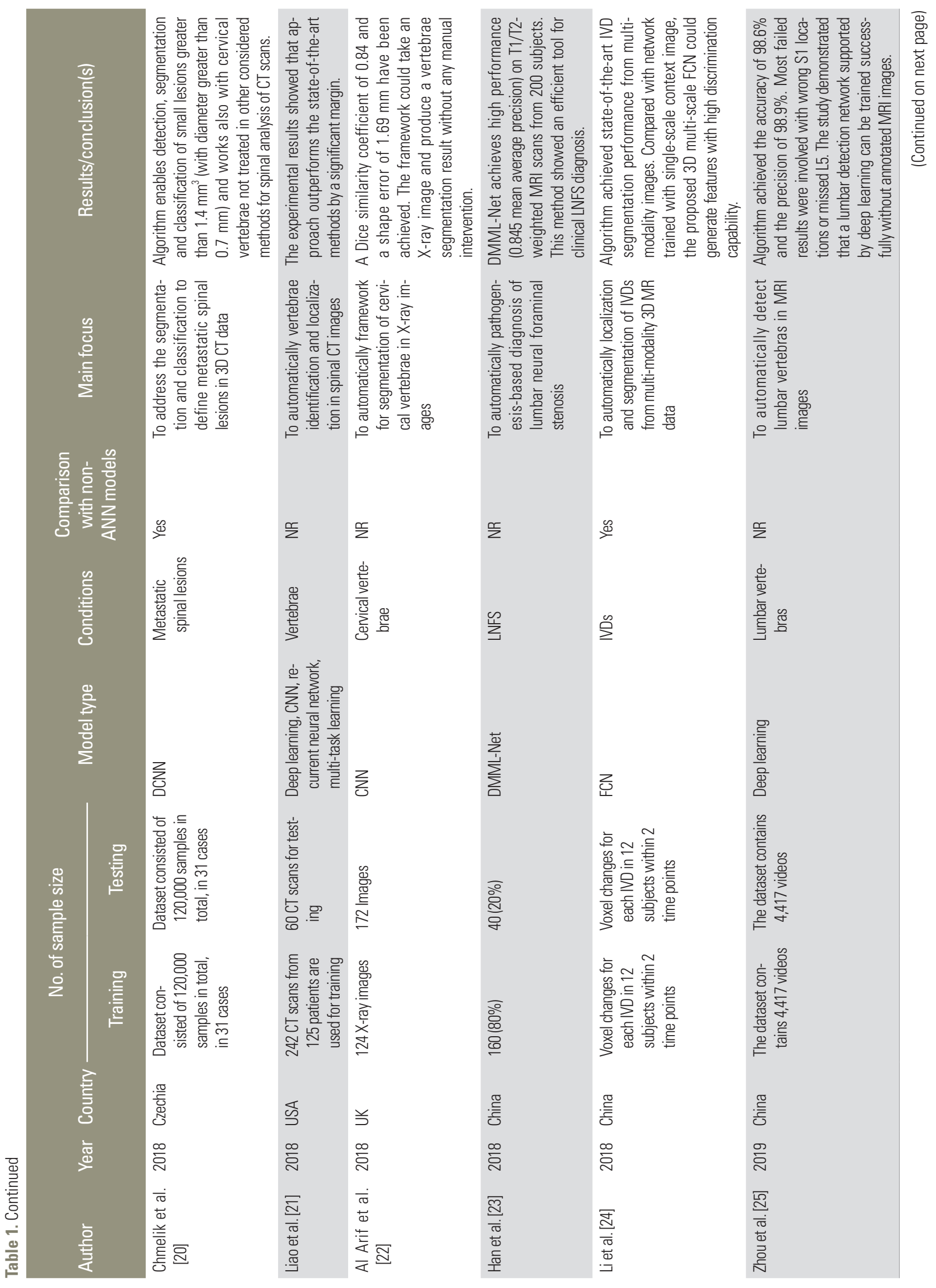




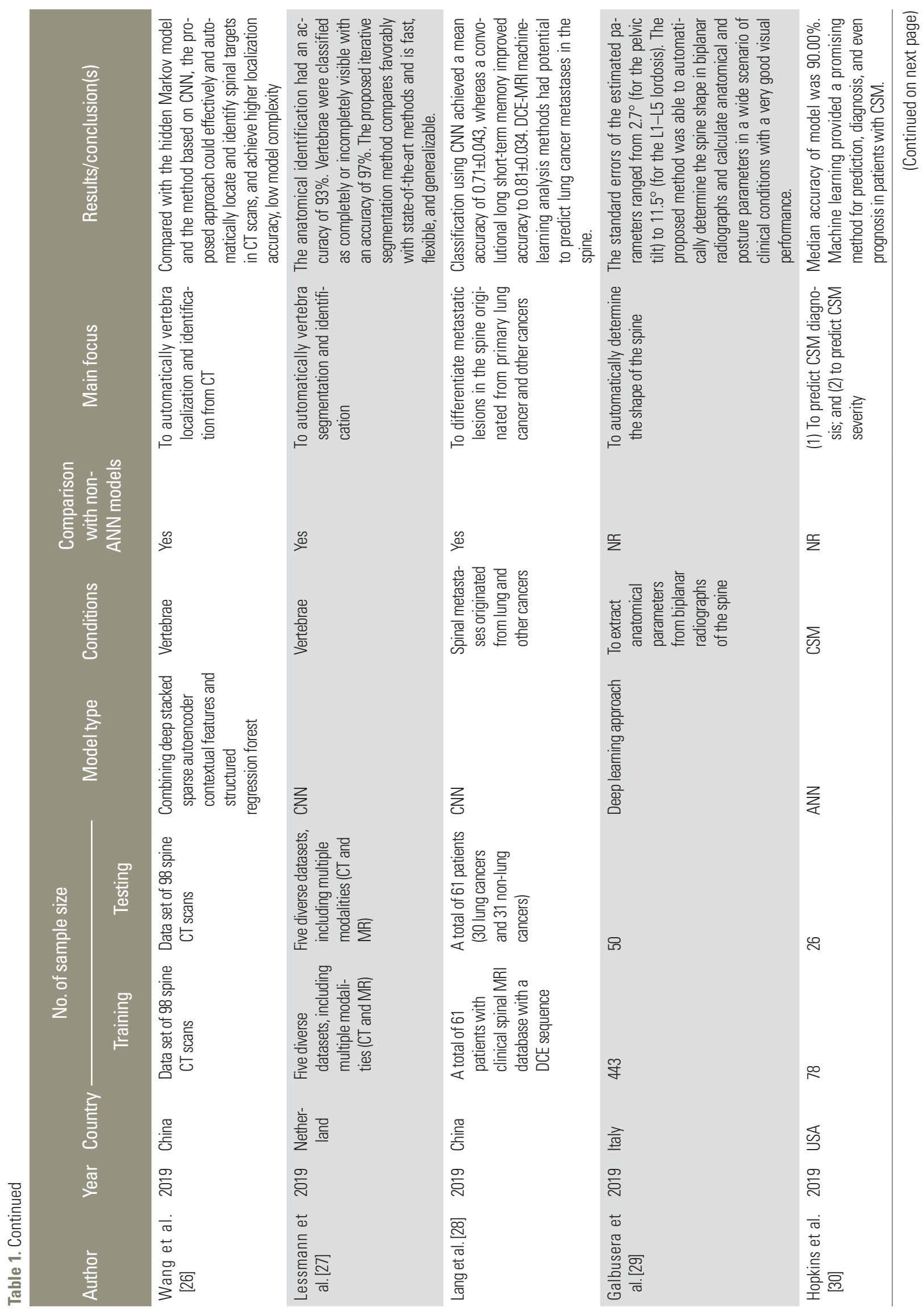




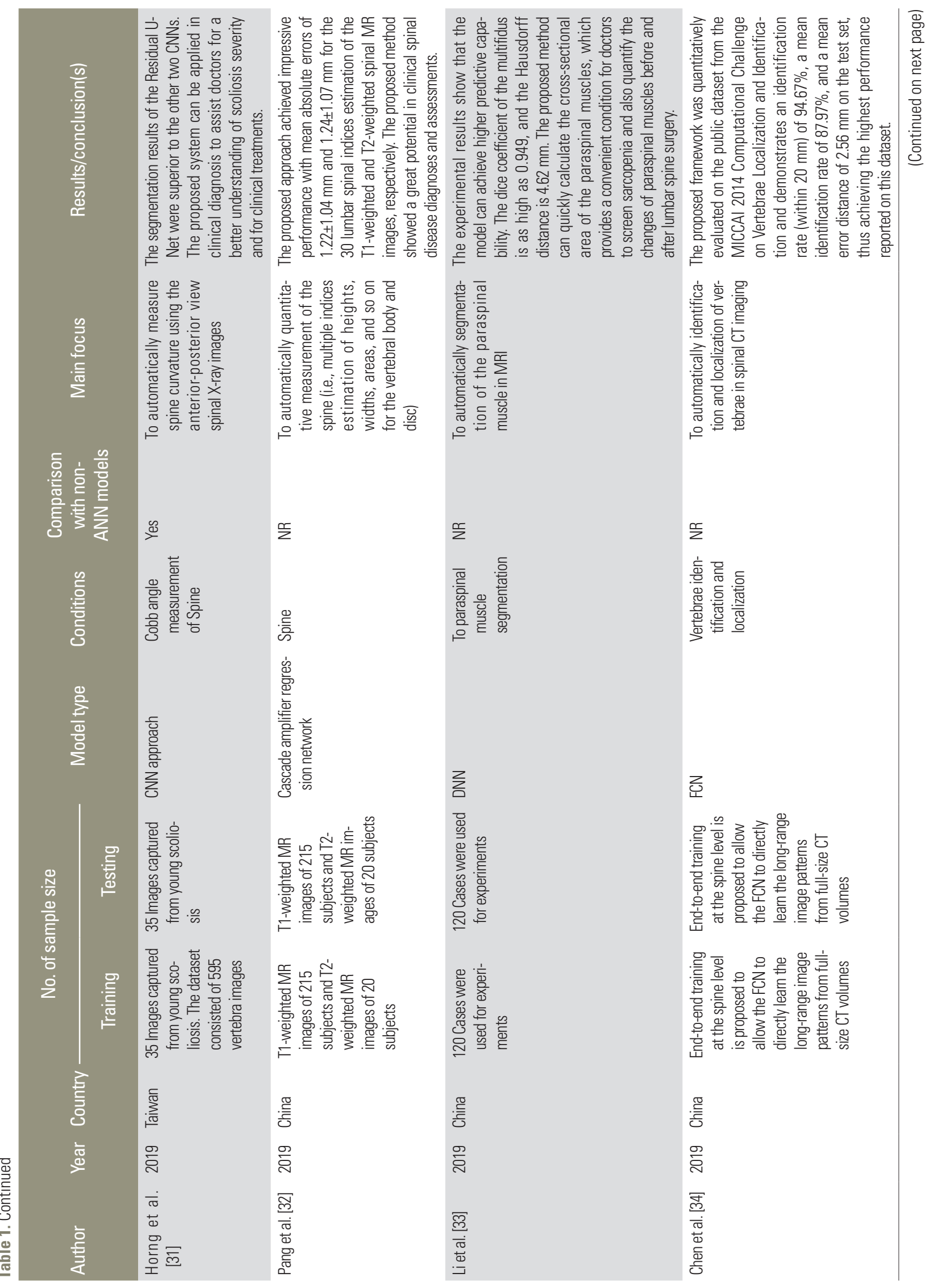




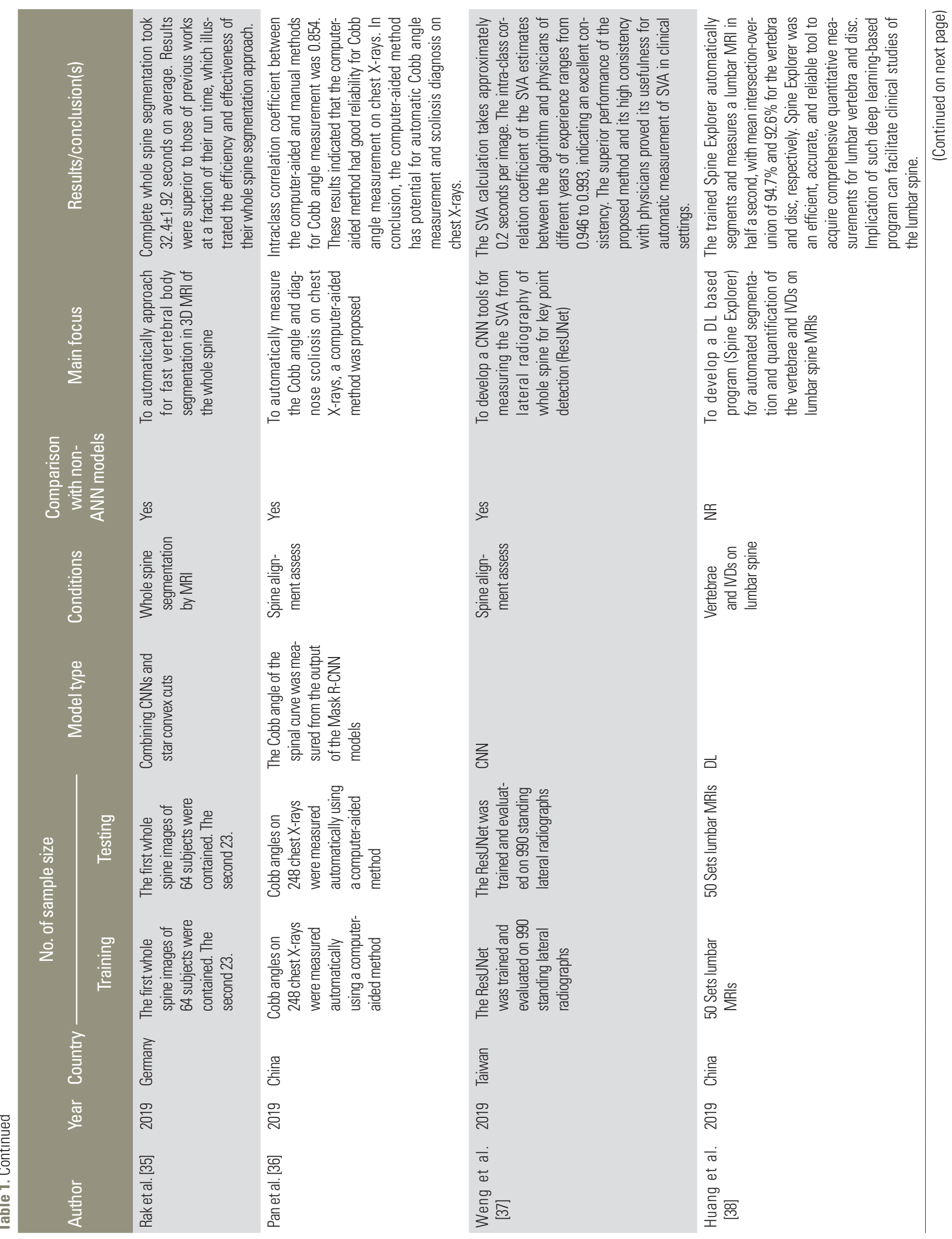




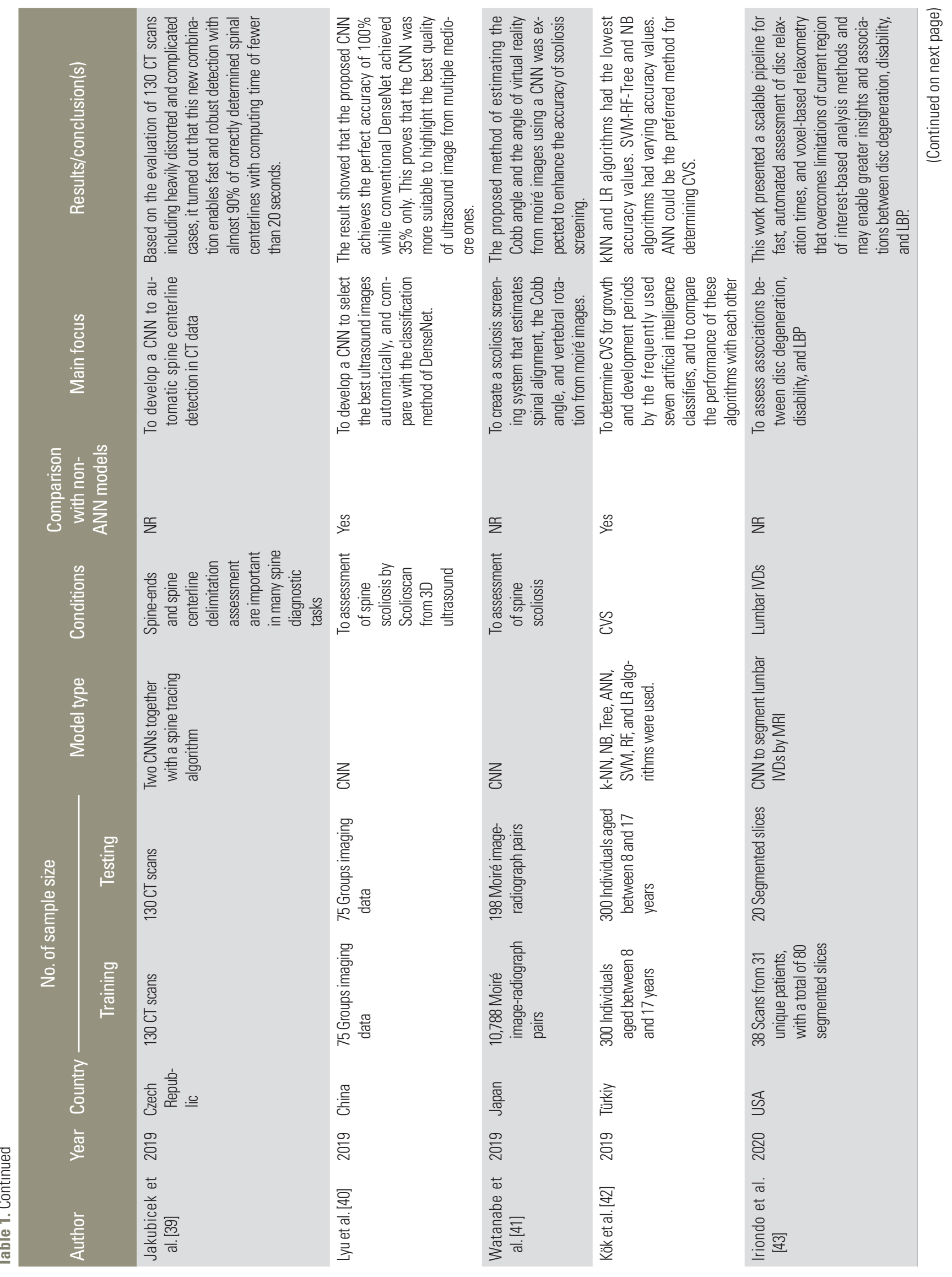




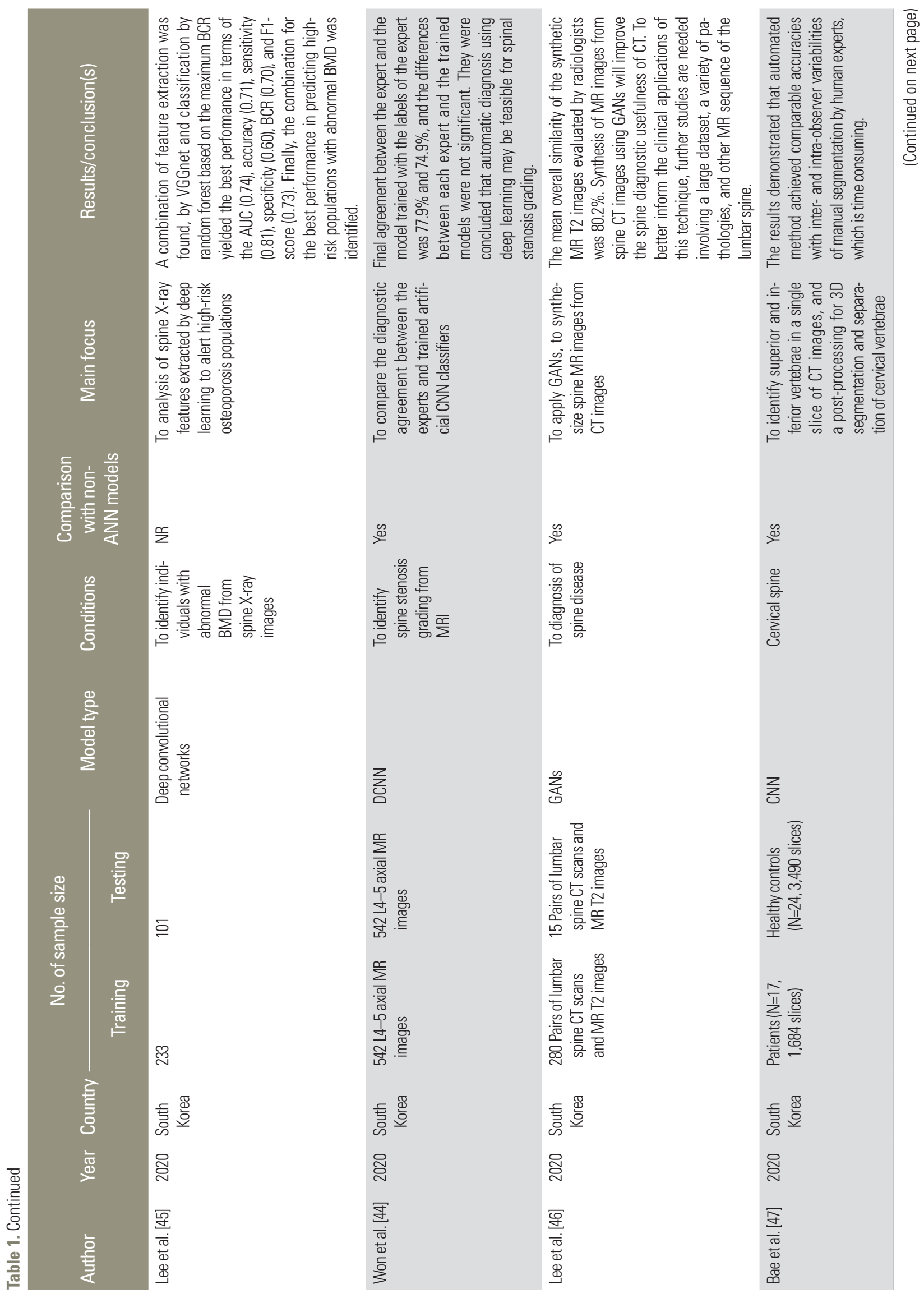




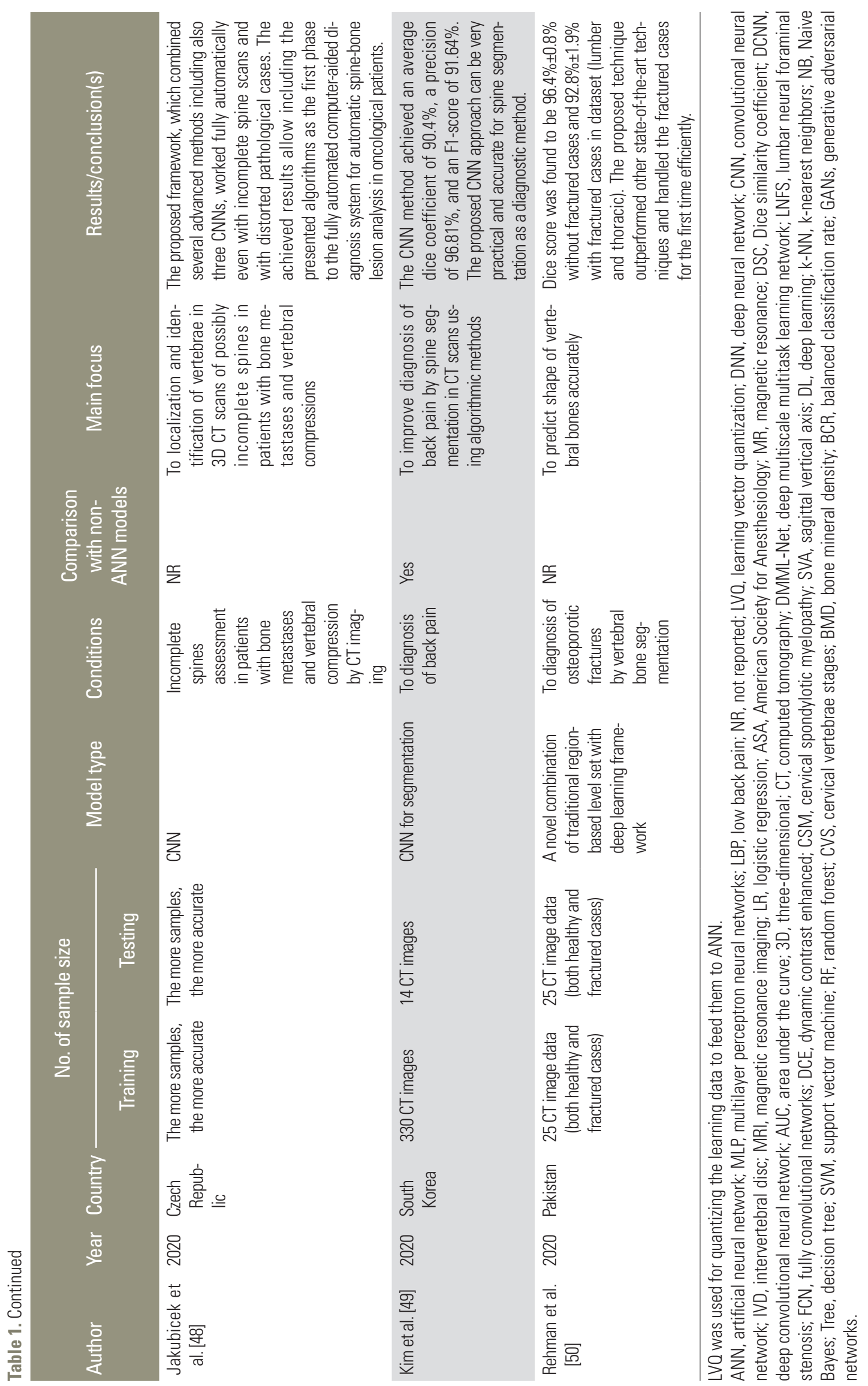


the commonly used clinical scoring methods [16]. A summary of the studies is shown in Table 2.

\section{Outcome prediction}

Table 3 summarizes the studies that used ANNs for outcome prediction. ANNs have been used to predict outcome in lumbar spinal stenosis (LSS) [3] and LDH [4], predict recurrent LDH [66], enhance surgical decision making for LSS [67], develop ANN algorithms for prediction of in-hospital and 90-day post-discharge mortality in spinal epidural abscess [68], predict non-routine discharge for patients undergoing surgery for lumbar disc disorders [69], assess vertebral strength and predict vertebral fracture risk in elderly patients [70], predict 30day readmission after posterior lumbar fusion [71], and predict surgical site infections after posterior spinal fusion [72].

\section{The use of artificial neural networks for the biome- chanical assessments of spinal diseases}

A clear understanding of biomechanical principles is important in the management of spinal disorders. There are ANNs studies focused on the biomechanics of spine via clarification of joint moments, spinal loads, and muscle forces [75-77]. Other than that, application of ANNs for optimization of the design of spinal pedicle screws [78], prediction of vertebral strength through machine learning models [70], determination of the consistency of the patient pain drawing in lumbar spine disease [73], prediction of low bone mineral density [74], recognition of low back pain patients from healthy population performed static standing tasks [79], estimation of three-dimensional whole-body posture, lumbosacral moments and spinal loads during load-handling activities [80] and automated tracking of lumbar vertebras with rotated bounding boxes in digitalized video fluoroscopic imaging, and motion and gait analysis [81] have been reported. These findings are summarized in Table 4.

\section{Discussion}

To the best of our knowledge, this is the first review devoted exclusively to an application of ANN in support of decision for management of spinal disease. Our findings offer a summary of relevant publications and a roadmap to guide future research related to the use of ANNs in spinal disease. Precisely, our findings showed that ANNs are powerful tools with the ability to improve understanding of predictive metrics, prognosis, diagnosis and biomechanical assessment in spinal diseases. Moreover, ANNs have shown consistent superiority over the traditional statistical approaches. In light of the continuous development of hardware and software methods, and advanced computational science and technology, wider consideration and broader application artificial intelligence in spinal disease is expected in the near future [2].

The number of publications on neural networks in spinal diseases has increased rapidly over the past few years, wherein a majority of the publications were in the domain of diagnosis of spinal disorders, followed by prognosis, prediction, and biomechanical for spinal applications. A number of ANN studies have focused on preoperative assessment, planning, intraoperative assistance and outcome prediction in spine surgery. Recently, Khor et al. [82] successfully developed a state-of-the-art use of a logistic regression (LR) model to predict the patient-reported outcomes in lumbar fusion surgery. They developed a clinical prediction tool model to determine the probabilities of improvement in function, back pain, and leg pain in lumbar fusion candidates at 1-year follow-up after surgery. This model showed a good accuracy in the validation cohorts. The same group also provided an online version of their prediction model for public use (https://becertain. shinyapps.io/lumbar_fusion_calculator/), where a clinician and/or patient can enter the individual demographics to predict a patient's likelihood of benefiting from a lumbar fusion procedure [83]. Besides, Karhade et al. [64] developed a machine learning tool for predicting prolonged postoperative opioid prescription in the patients undergoing LDH surgery (https://sorg-apps.shinyapps.io/ lumbardiscopioid/). It is worth mentioned that preoperative prediction of opioid use could improve the risk stratification, shared decision-making, and patient counseling before LDH surgery [84]. In addition, Karhade et al. [61] developed a machine learning tool to automatically predict 90-day and 1-year mortality in spinal metastatic disease (https://sorg-apps.shinyapps.io/spinemetssurvival/) [85]. Meanwhile, the same group also developed a machine learning algorithm for predicting discharge disposition after elective inpatient surgery for lumbar disc disease [55], the model is available at https://sorg-apps.shinyapps. io/discdisposition/ [86]. Furthermore, there is also an 


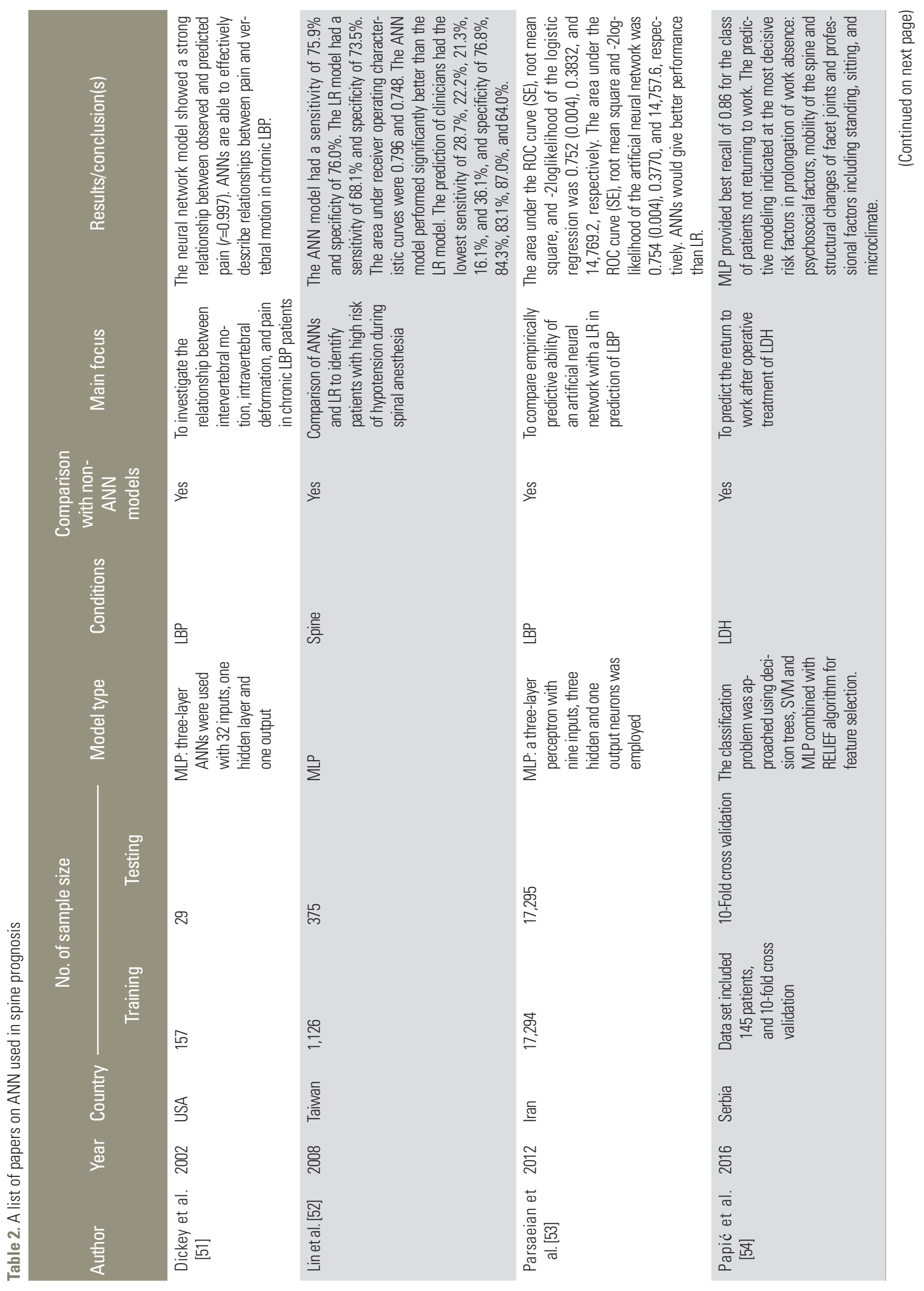




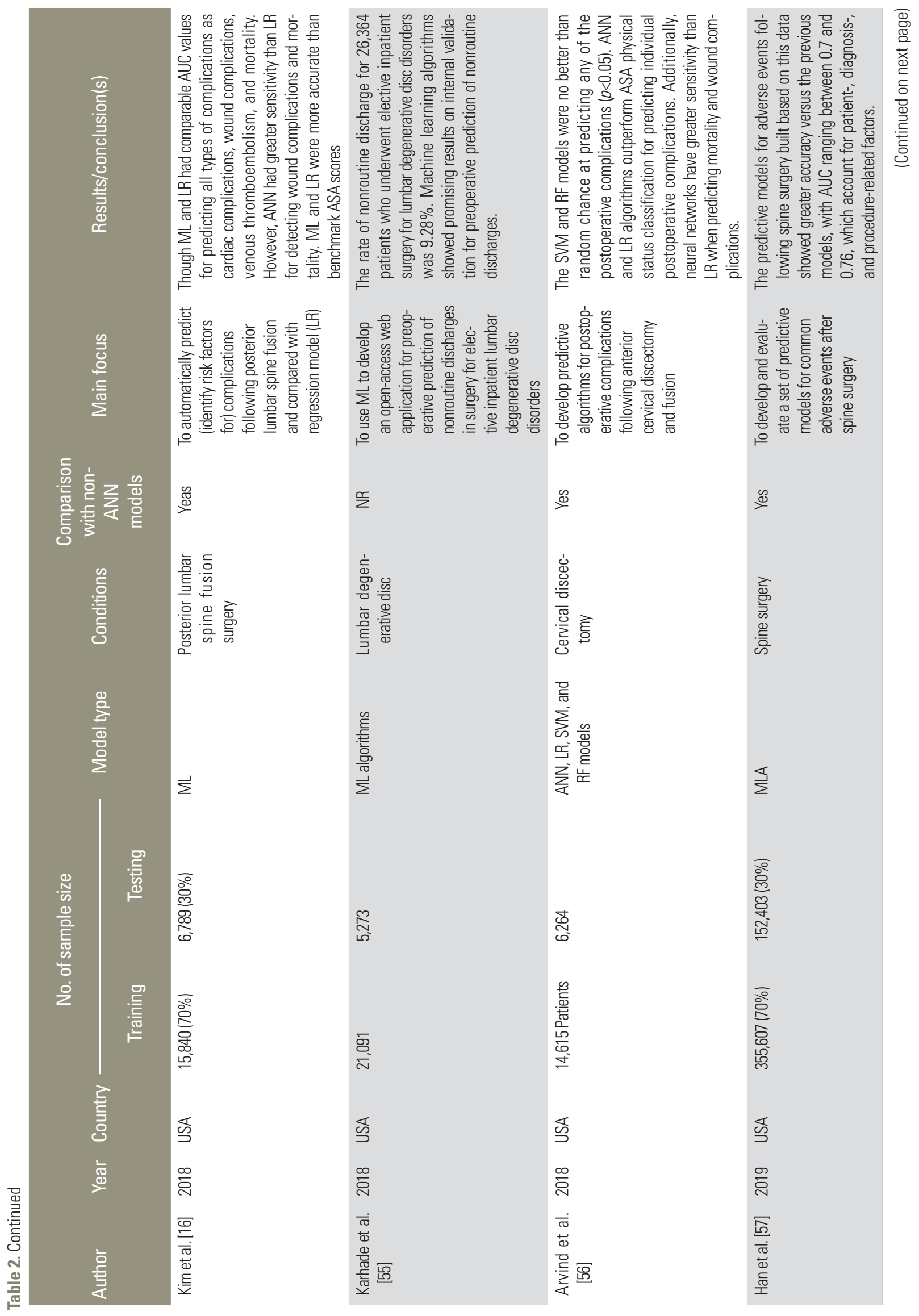




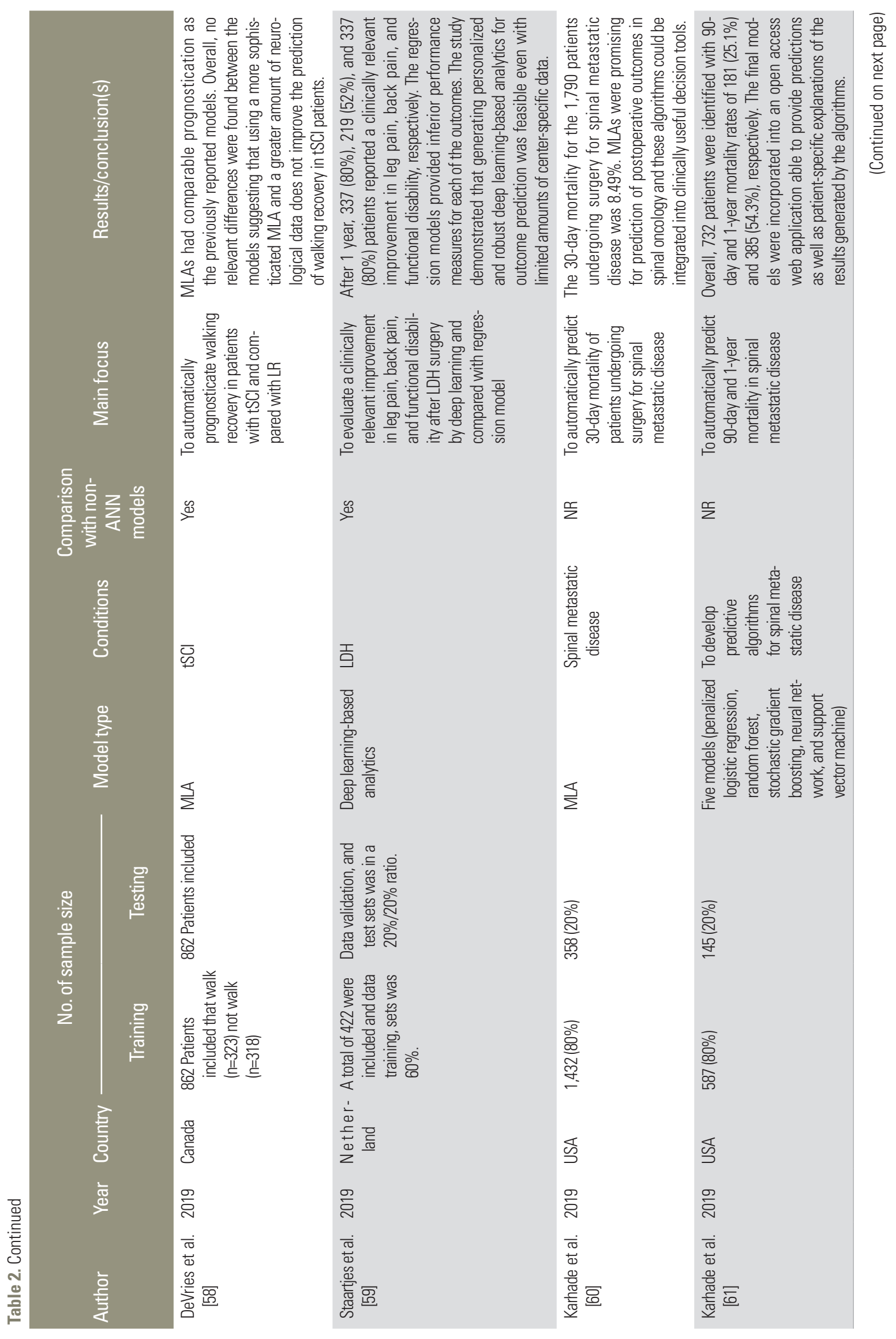




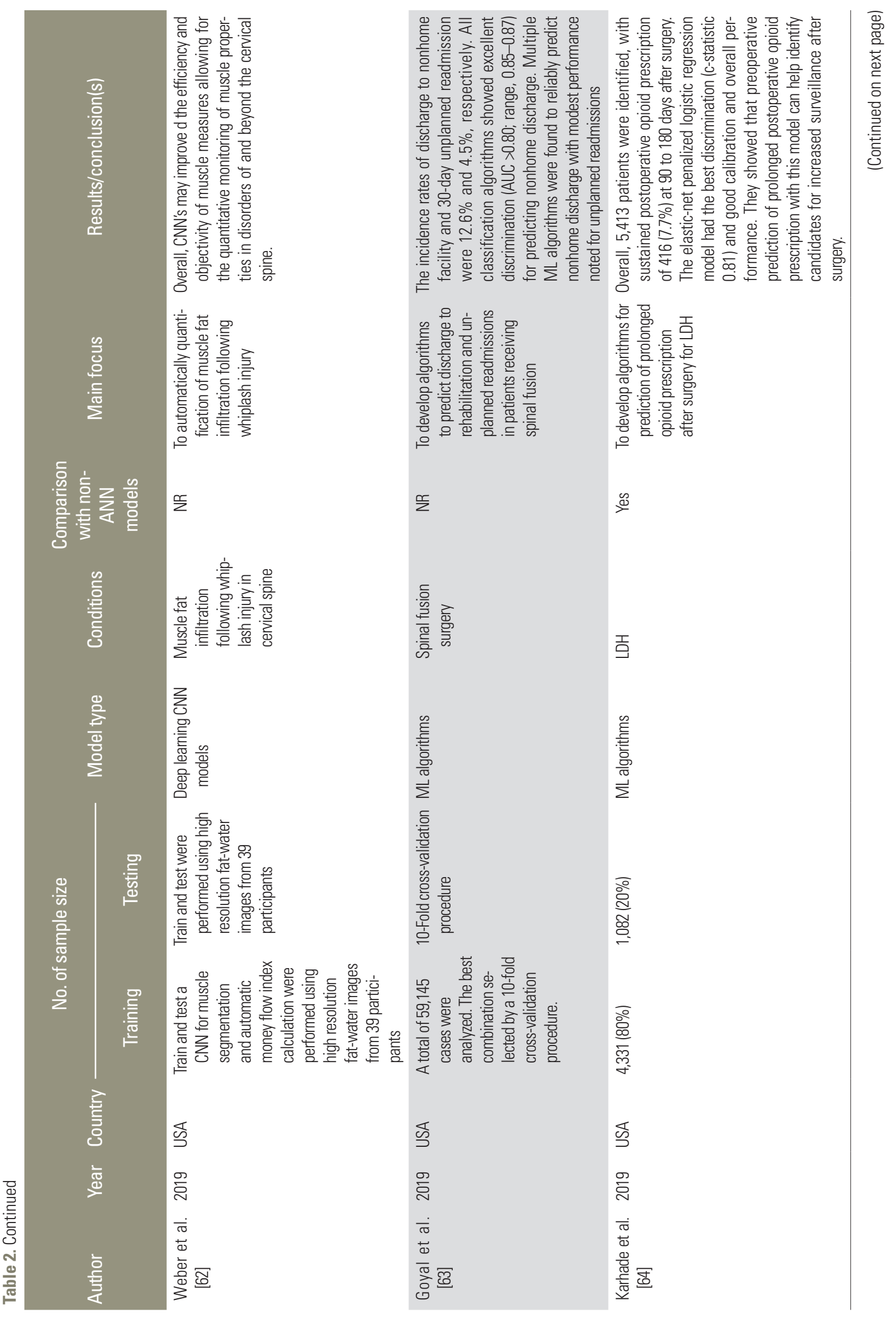




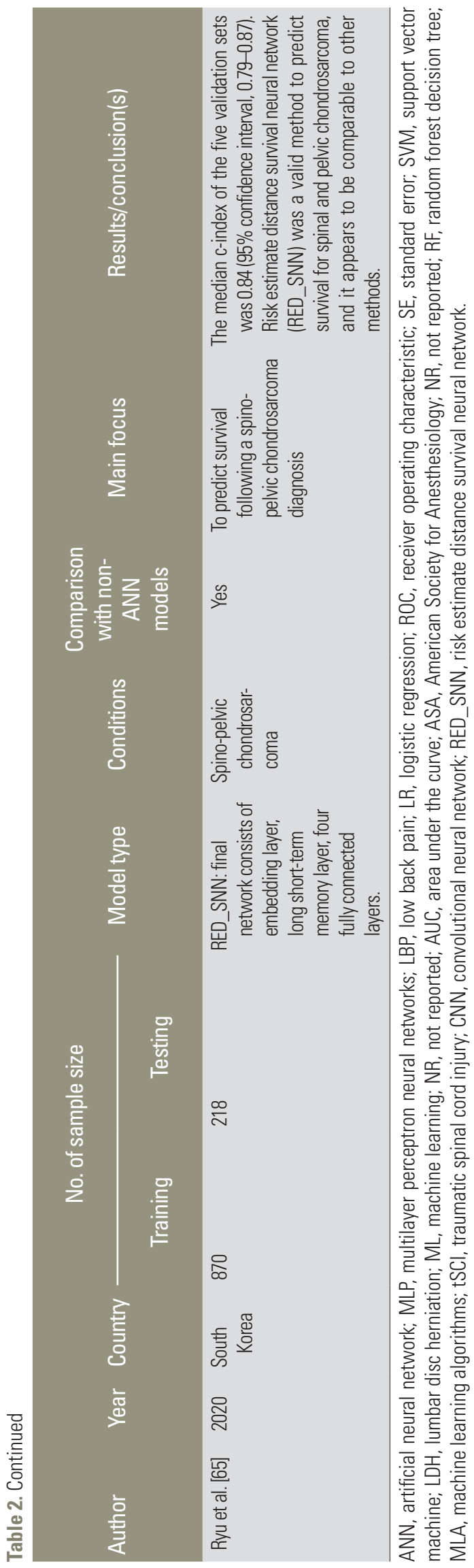

application at https://sorg-apps.shinyapps.io/spinemets/ [87] which allows prediction of 30-day mortality after surgery for spinal metastatic disease [60]. Nonetheless, these machine learning tools are fitted for general educational purposes and they are not capable of substituting the professional medical advice, consultation, diagnosis, or treatment [82-87]. One might inquire about how the ANNs can assist in the clinical decision-making process? Spine neural network tools will never replace human experts, but it helps in screening and can be used by the experts to validate their diagnosis, prognosis, and prediction. More importantly, ANNs can be used to identify the variables that experts may not observe, thus enhancing the diagnostic acumen of experts. As aforementioned, the currently available web applications are not a good fit with the clinical practice setting. However, development of this software tool is a prerequisite for an international consultancy group to satisfy the diverse needs as randomized clinical trials (RCT) data exists that specifically examines this tool. Nevertheless, ANNs will never replace human expert decision-making, but it can assist in validating the routine decision-making process [2].

Few neural network studies have focused on medical imaging analysis. There are a wide variety of medical imaging modalities and magnetic resonance imaging (MRI) is majorly applied for clinical diagnosis and prognosis. Recently, some studies have demonstrated successful application of artificial intelligence algorithms for spine medical image segmentation [17,19,20,22,24,27,3 $3,35,38,47,49$ ], computer-aided spine diagnosis [84-87], and disease detection and classification $[10,45]$. In other words, spinal images could be analyzed, processed, and categorized by using neural network. By selecting a suitable training set and learning process, neural networks is appropriate for recognition of unusual images [88]. Artificial intelligence will play a vital role in the development of medical image analysis methods. However, deep learning architecture requires a large amount of training data and computational power. Currently, there is also a rising interest with respect to the digital image analysis solutions with artificial intelligence for clinical applications. Such applications aim to increase diagnostic and prognostic accuracy, reliability, and efficiency by enabling quantitative image analysis. For instance, Oxford SpineNet software system, a machine learning based system for automated analysis of spinal T2 MRI scans acquired from a DICOM (Digital Imaging and Communications in Medicine) file, 


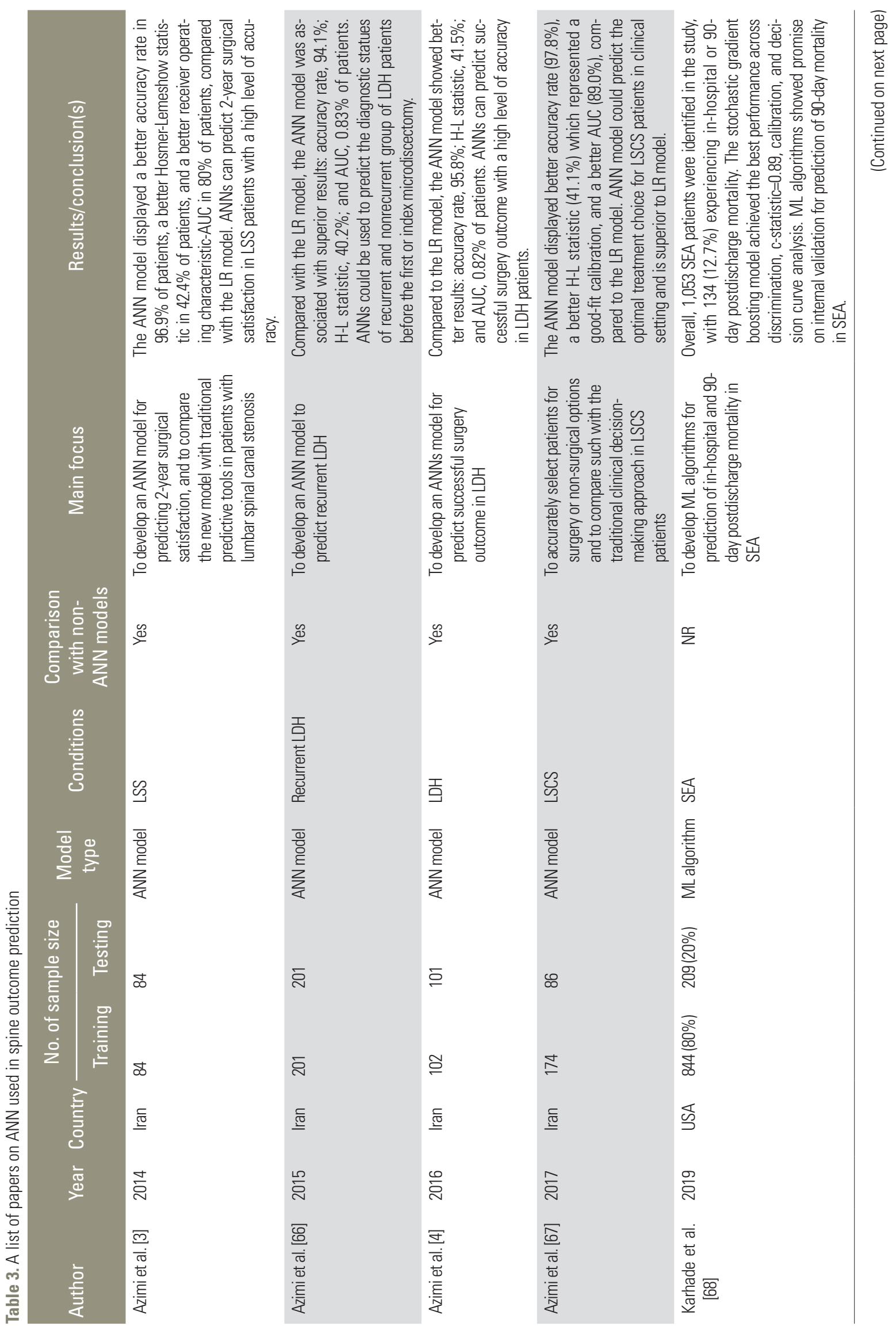




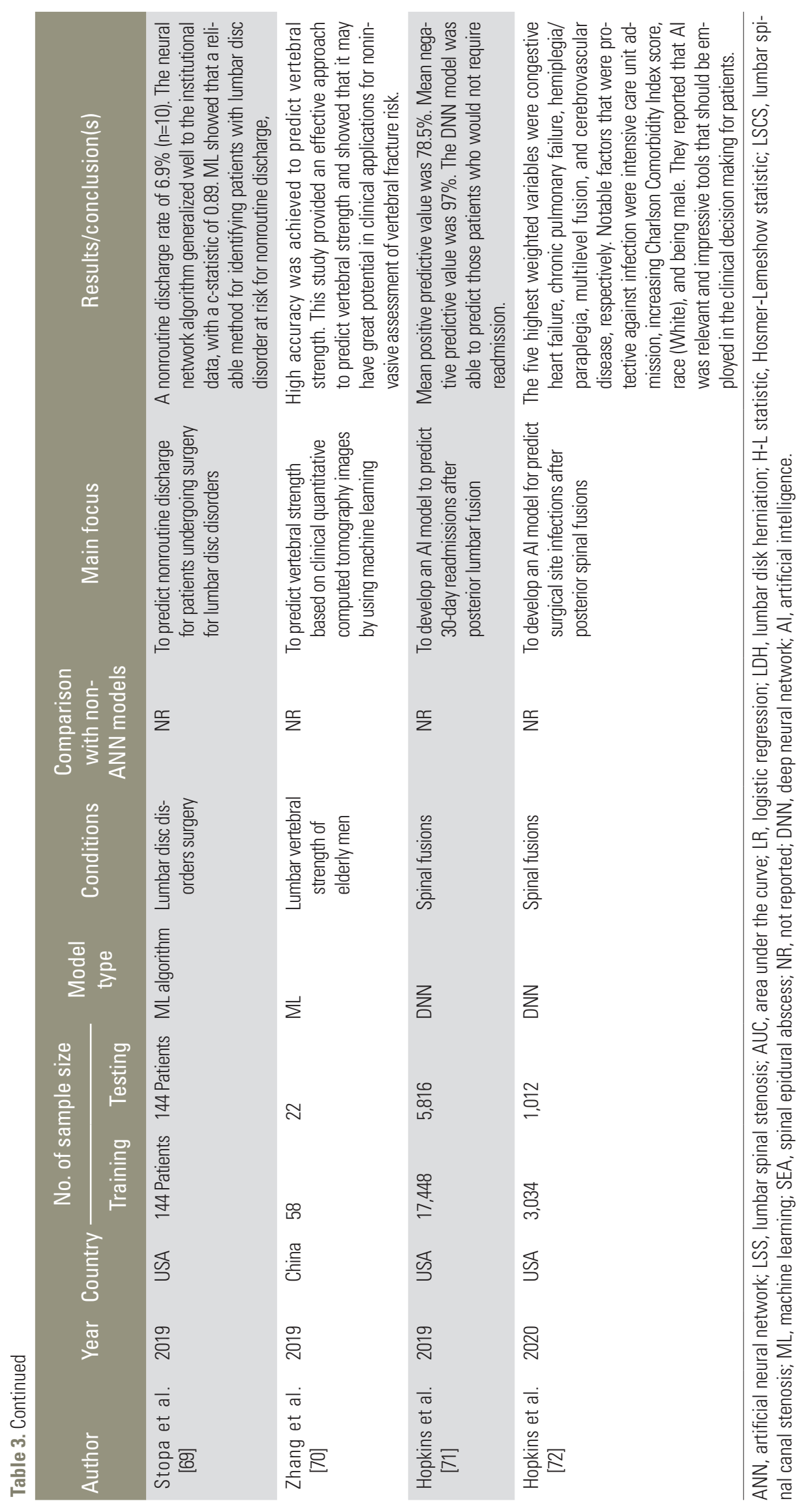




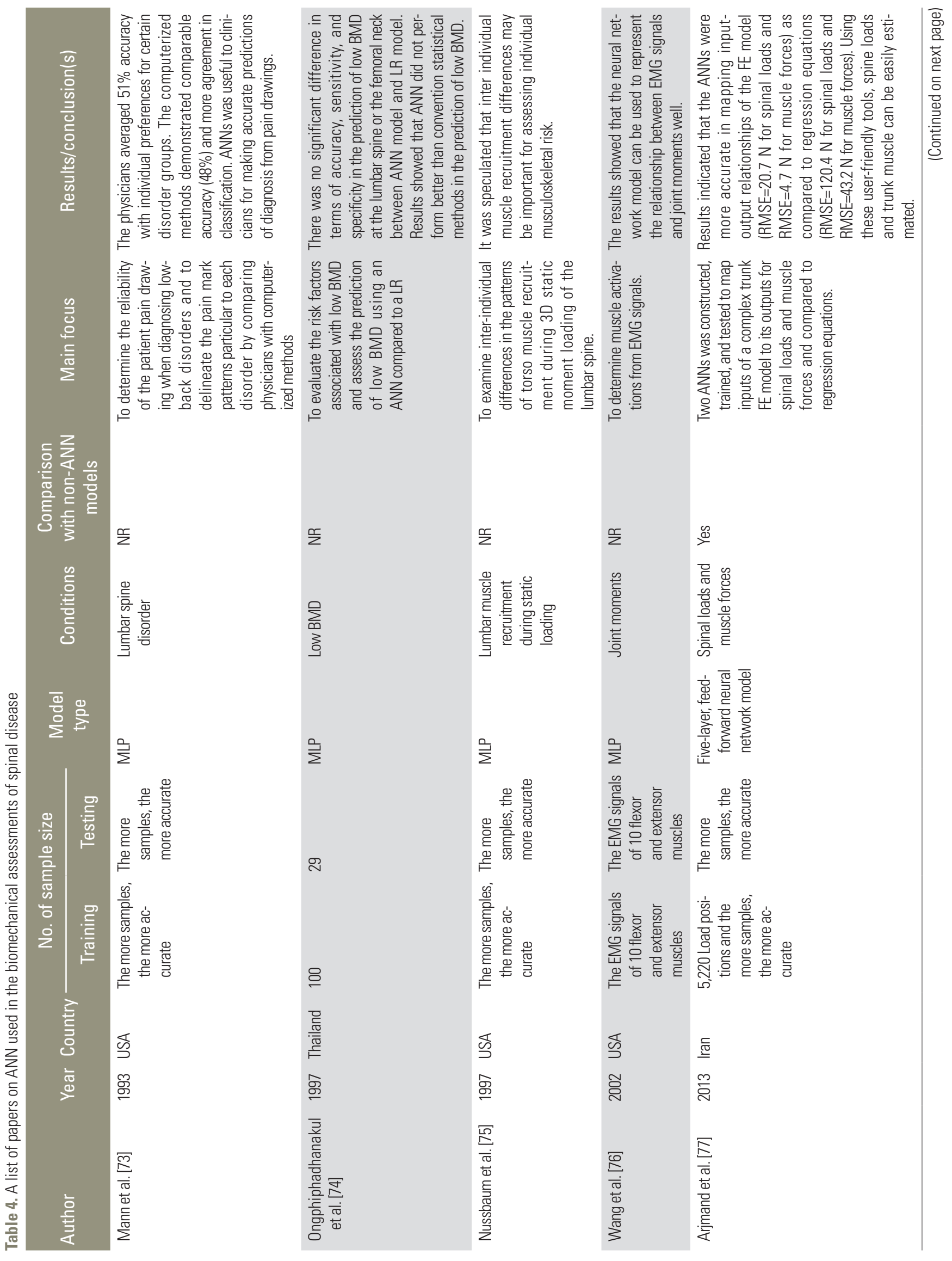




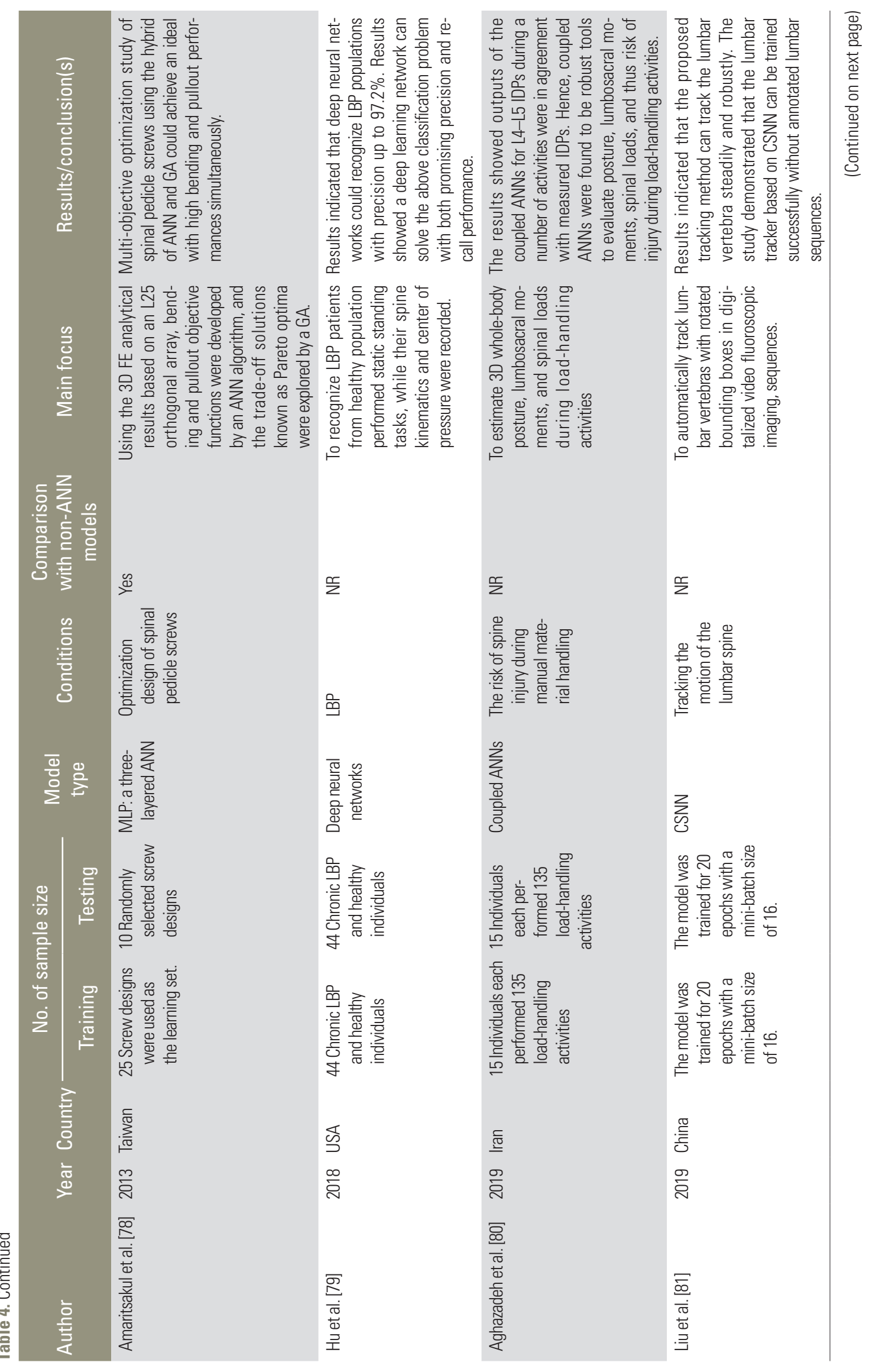




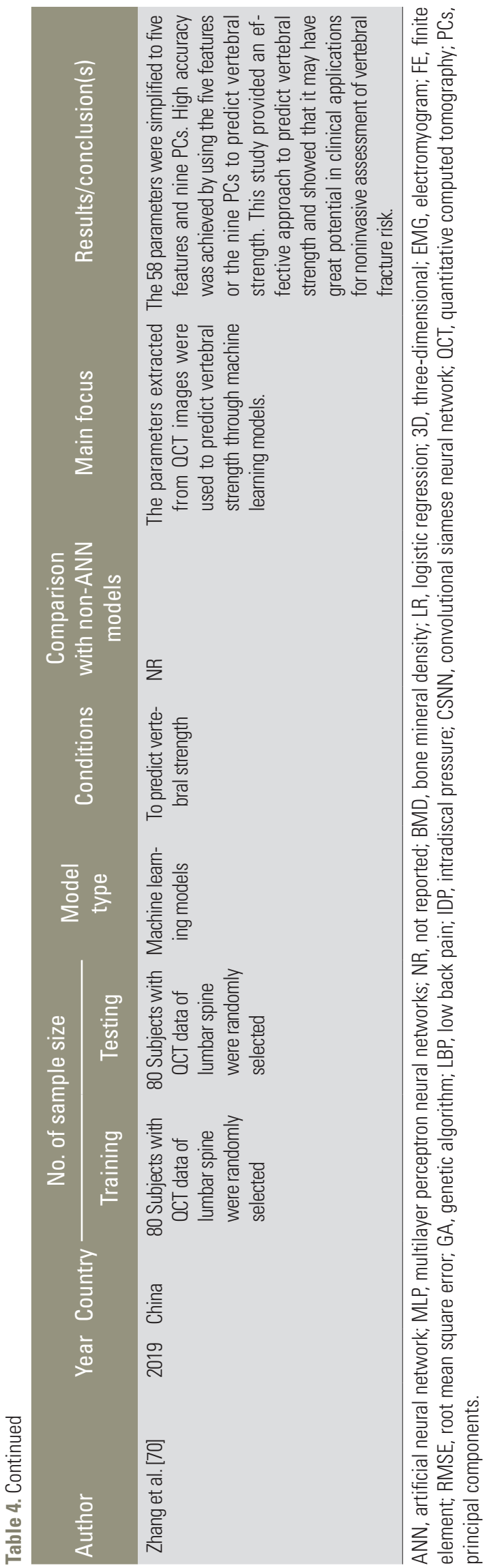

introduced by Jamaludin et al. [14,89] helps in clinical and algorithmic research [90]. This system can extract a wide range of relevant measurements from magnetic resonance (MR) images automatically including Pffirrmann grades, Modic changes, spinal stenosis, grading LSS into four grades, and disc herniation. The software needs to be able to learn without human input and classify multiple radiological features simultaneously. Therefore, the SpineNet software system adopts a neural network which allows it to learn and classify multiple scores at the same time. The SpineNet software tool is available online at http:// zeus.robots.ox.ac.uk/spinenet/. Meanwhile, the authors stated that this is neither a diagnostics tool nor a medical device; hence, it should be used for research only [90]. In the future, ANN tools for the spinal workflow may help to empower the spine surgeon in management of spinal diseases, positively engage the patients, reduce the probability of error and improve spine surgeon efficiency.

ANNs have been effectively applied for biomechanics of spine, such as estimation of loads and stresses [80], estimation of the material properties of biological tissues [74], and analysis of the motion and gait [81]. A clear understanding of biomechanical principles is vital for the treatment of spinal disorders [91]. In view of the direct and invasive in vivo measurements of spinal loads and muscle forces, researchers have decided to apply ANNs for spinal biomechanical assessment by using different possible models [77]. Yet, the use of artificial intelligence techniques in clinical biomechanics of the spine is still in its infancy. However, continued assessment using ANN methods and understanding of mathematical and bioengineering principles will ensure the discovery of new solutions and more effective ways of helping spine patients, especially spine surgery [75-78]. In general, at the present time, there is no clinical application available for biomechanics of spine. Nonetheless, the aforementioned studies clearly demonstrate the potential of neural network in this arena.

There was considerable heterogeneity in the modeling methods used. Studies varied with regard to the inclusion criteria, input and output variables used, machine learning techniques applied, and models performance for the evaluation of the four categories of issues discussed above. Yet, LR, neural networks, and support vector machines (SVM) are the most commonly applied computer-based algorithms. However, these algorithms are developing and require further improvement. As an 
example, the following machine learning techniques are the most recommended to be employed: Bi-directional Long Short-Term Memory, Deep Learning and Neural Network, Extreme Machine Learning, SVM and TBasts, Random Forest, and Boosting [92]. On the other hand, the comparison of different neural network algorithms could assist in the selection of the best model for making sure that it has the best performance. However, it is also crucial to consider the advantages and disadvantages of these algorithms based on the dataset details and planed application to make the best choice [92]. Hence, there is a need for method standardization in order to apply ANNs spine clinical practice.Although the current research highlights the promise and potential of neural networks in spine disorders, there is continuing debate whether neural networks could or should be used in routine clinical practice. At present, there is a paucity of literature on the clinical application of neural networks in spine clinical decision-making. In addition, there are no specific consensus recommendations for designing an optimal ANN for clinical practice [2]. Hence, in order to develop an ANN tool for spine clinical practice, it is recommended that an international center need to consider the following subjects: (1) to create a multidisciplinary team of spine clinicians, engineers and data scientists to evaluate ANN tools; (2) to design methods of data collection for training and testing, and standardization for each spinal disorders; (3) to select the best neural network algorithm; (4) to develop and improve user-friendly software environment; (5) to validate ("model testing"); (6) to assess ANNs as tools for performing RCT; and (7) to provide up-to-date ANN models which could handle higher patient data (big data) entry for augmenting augment decision-making efficacy [2]. In general, the preliminary results from tools are satisfactory. However, it still requires many efforts to make the ANN tools more accurate and to make the idea practically achievable.

ANN technology has been attracting substantial attention in spinal disease, but there are challenges to be implemented in clinical setting. Several limitations exist in most of the studies on ANNs in spinal diseases, including largely heterogeneous study design, data analysis, modeling technique, training and testing features applied, algorithms employed, and end points. Hence, a focused synthesis of the literature cannot be provided. In addition, the search strategy was limited to the keywords in the titles or abstracts of publications. Thus, we might have missed some papers. Secondly, this work restricted the query search for articles in PubMed. Thirdly, non-English publications were not considered in this study. We believe that the research regarding the application of ANNs in spinal diseases have also been published in other languages. Fourthly, most ANNs algorithms in these studies were validated with their dataset, it may be lack of external validation and generalizability of their results. Fifthly, some studies did not compare ANNs with conventional statistical analysis; hence, a comparison between any two models is limited [2].

\section{Conclusions}

The evidence suggests that ANNs can be successfully used for spinal disease to manage its diagnosis, prognosis and outcome prediction. Further ANNs algorithm retraining, generalizability of models, data standardization in neural networks, and focus on the application of ANNs as a tool in clinical spine practice, will augment decision-making efficacy.

\section{Conflict of Interest}

No potential conflict of interest relevant to this article was reported.

\section{Acknowledgments}

The authors thank the staff of the Neurosurgery Unit at Imam-Hossain Hospital, Tehran, Iran.

\section{References}

1. Azimi P, Mohammadi HR. Predicting endoscopic third ventriculostomy success in childhood hydrocephalus: an artificial neural network analysis. J Neurosurg Pediatr 2014;13:426-32.

2. Azimi P, Mohammadi HR, Benzel EC, Shahzadi S, Azhari S, Montazeri A. Artificial neural networks in neurosurgery. J Neurol Neurosurg Psychiatry 2015;86:251-6.

3. Azimi P, Benzel EC, Shahzadi S, Azhari S, Mohammadi HR. Use of artificial neural networks to predict surgical satisfaction in patients with lumbar spinal 
canal stenosis: clinical article. J Neurosurg Spine 2014;20:300-5.

4. Azimi P, Benzel EC, Shahzadi S, Azhari S, Mohammadi HR. The prediction of successful surgery outcome in lumbar disc herniation based on artificial neural networks. J Neurosurg Sci 2016;60:173-7.

5. Nolting J. Developing a neural network model for health care. AMIA Annu Symp Proc 2006;2006:1049.

6. Bishop JB, Szpalski M, Ananthraman SK, McIntyre DR, Pope MH. Classification of low back pain from dynamic motion characteristics using an artificial neural network. Spine (Phila Pa 1976) 1997;22:29918.

7. Jaremko JL, Poncet P, Ronsky J, et al. Estimation of spinal deformity in scoliosis from torso surface cross sections. Spine (Phila Pa 1976) 2001;26:1583-91.

8. Stanley RJ, Long R. A radius of curvature-based approach to cervical spine vertebra image analysis. Biomed Sci Instrum 2001;37:385-90.

9. Liszka-Hackzell JJ, Martin DP. Categorization and analysis of pain and activity in patients with low back pain using a neural network technique. J Med Syst 2002;26:337-47.

10. Lin $\mathrm{H}$. Identification of spinal deformity classification with total curvature analysis and artificial neural network. IEEE Trans Biomed Eng 2008;55:376-82.

11. Sari M, Gulbandilar E, Cimbiz A. Prediction of low back pain with two expert systems. J Med Syst 2012;36:1523-7.

12. Veronezi CC, de Azevedo Simoes PW, Dos Santos $\mathrm{RL}$, et al. Computational analysis based on artificial neural networks for aiding in diagnosing osteoarthritis of the lumbar spine. Rev Bras Ortop 2015;46:1959.

13. Zhang J, Li H, Lv L, Zhang Y. Computer-aided cobb measurement based on automatic detection of vertebral slopes using deep neural network. Int J Biomed Imaging 2017;2017:9083916.

14. Jamaludin A, Lootus M, Kadir T, et al. Issls Prize in Bioengineering Science 2017: automation of reading of radiological features from magnetic resonance images (MRIs) of the lumbar spine without human intervention is comparable with an expert radiologist. Eur Spine J 2017;26:1374-83.

15. Wang J, Fang Z, Lang N, Yuan H, Su MY, Baldi P. A multi-resolution approach for spinal metastasis detection using deep Siamese neural networks. Comput
Biol Med 2017;84:137-46.

16. Kim JS, Merrill RK, Arvind V, et al. Examining the ability of artificial neural networks machine learning models to accurately predict complications following posterior lumbar spine fusion. Spine (Phila Pa 1976) 2018;43:853-60.

17. Kim S, Bae WC, Masuda K, Chung CB, Hwang D. Fine-grain segmentation of the intervertebral discs from MR spine images using deep convolutional neural networks: BSU-Net. Appl Sci (Basel) 2018;8:1656.

18. Kim K, Kim S, Lee YH, Lee SH, Lee HS, Kim S. Performance of the deep convolutional neural network based magnetic resonance image scoring algorithm for differentiating between tuberculous and pyogenic spondylitis. Sci Rep 2018;8:13124.

19. Han Z, Wei B, Mercado A, Leung S, Li S. Spine-GAN: semantic segmentation of multiple spinal structures. Med Image Anal 2018;50:23-35.

20. Chmelik J, Jakubicek R, Walek P, et al. Deep convolutional neural network-based segmentation and classification of difficult to define metastatic spinal lesions in 3D CT data. Med Image Anal 2018;49:76-88.

21. Liao H, Mesfin A, Luo J. Joint vertebrae identification and localization in spinal CT images by combining short- and long-range contextual information. IEEE Trans Med Imaging 2018;37:1266-75.

22. Al Arif SMMR, Knapp K, Slabaugh G. Fully automatic cervical vertebrae segmentation framework for $\mathrm{X}$-ray images. Comput Methods Programs Biomed 2018;157:95-111.

23. Han Z, Wei B, Leung S, Nachum IB, Laidley D, Li S. Automated pathogenesis-based diagnosis of lumbar neural foraminal stenosis via deep multiscale multitask learning. Neuroinformatics 2018;16:325-37.

24. Li X, Dou Q, Chen H, et al. 3D multi-scale FCN with random modality voxel dropout learning for intervertebral disc localization and segmentation from multi-modality MR images. Med Image Anal 2018;45:41-54

25. Zhou Y, Liu Y, Chen Q, Gu G, Sui X. Automatic lumbar MRI detection and identification based on deep learning. J Digit Imaging 2019;32:513-20.

26. Wang X, Zhai S, Niu Y. Automatic vertebrae localization and identification by combining deep SSAE contextual features and structured regression forest. J Digit Imaging 2019;32:336-48.

27. Lessmann N, van Ginneken B, de Jong PA, Isgum 
I. Iterative fully convolutional neural networks for automatic vertebra segmentation and identification. Med Image Anal 2019;53:142-55.

28. Lang N, Zhang Y, Zhang E, et al. Differentiation of spinal metastases originated from lung and other cancers using radiomics and deep learning based on DCE-MRI. Magn Reson Imaging 2019;64:4-12.

29. Galbusera F, Niemeyer F, Wilke HJ, et al. Fully automated radiological analysis of spinal disorders and deformities: a deep learning approach. Eur Spine J 2019;28:951-60.

30. Hopkins BS, Weber KA 2nd, Kesavabhotla K, Paliwal M, Cantrell DR, Smith ZA. Machine learning for the prediction of cervical spondylotic myelopathy: a post hoc pilot study of 28 participants. World Neurosurg 2019;127:e436-42.

31. Horng MH, Kuok CP, Fu MJ, Lin CJ, Sun YN. Cobb angle measurement of spine from X-ray images using convolutional neural network. Comput Math Methods Med 2019;2019:6357171.

32. Pang S, Su Z, Leung S, et al. Direct automated quantitative measurement of spine by cascade amplifier regression network with manifold regularization. Med Image Anal 2019;55:103-15.

33. Li H, Luo H, Liu Y. Paraspinal muscle segmentation based on deep neural network. Sensors (Basel) 2019;19:2650.

34. Chen Y, Gao Y, Li K, Zhao L, Zhao J. vertebrae identification and localization utilizing fully convolutional networks and a hidden Markov model. IEEE Trans Med Imaging 2020;39:387-99.

35. Rak M, Steffen J, Meyer A, Hansen C, Tonnies KD. Combining convolutional neural networks and star convex cuts for fast whole spine vertebra segmentation in MRI. Comput Methods Programs Biomed 2019;177:47-56.

36. Pan Y, Chen Q, Chen T, et al. Evaluation of a computer-aided method for measuring the Cobb angle on chest X-rays. Eur Spine J 2019;28:3035-43.

37. Weng CH, Wang CL, Huang YJ, et al. Artificial intelligence for automatic measurement of sagittal vertical axis using ResUNet framework. J Clin Med 2019;8:1826.

38. Huang J, Shen H, Wu J, et al. Spine Explorer: a deep learning based fully automated program for efficient and reliable quantifications of the vertebrae and discs on sagittal lumbar spine MR images. Spine J
2020;20:590-9.

39. Jakubicek R, Chmelik J, Ourednicek P, Jan J. Deeplearning-based fully automatic spine centerline detection in CT data. Conf Proc IEEE Eng Med Biol Soc 2019;2019:2407-10.

40. Lyu J, Ling SH, Banerjee S, et al. 3D ultrasound spine image selection using convolution learning-torank algorithm. Conf Proc IEEE Eng Med Biol Soc 2019;2019:4799-802.

41. Watanabe K, Aoki Y, Matsumoto M. An application of artificial intelligence to diagnostic imaging of spine disease: estimating spinal alignment from moire images. Neurospine 2019;16:697-702.

42. Kok H, Acilar AM, Izgi MS. Usage and comparison of artificial intelligence algorithms for determination of growth and development by cervical vertebrae stages in orthodontics. Prog Orthod 2019;20:41.

43. Iriondo C, Pedoia V, Majumdar S. Lumbar intervertebral disc characterization through quantitative MRI analysis: an automatic voxel-based relaxometry approach. Magn Reson Med 2020 Feb 14 [Epub]. https://doi.org/10.1002/mrm.28210.

44. Won D, Lee HJ, Lee SJ, Park SH. Spinal stenosis grading in magnetic resonance imaging using deep convolutional neural networks. Spine (Phila Pa 1976) 2020 Jan 20 [Epub]. https://doi.org/10.1097/ BRS.0000000000003384.

45. Lee S, Choe EK, Kang HY, Yoon JW, Kim HS. The exploration of feature extraction and machine learning for predicting bone density from simple spine $\mathrm{X}$-ray images in a Korean population. Skeletal Radiol 2020;49:613-8.

46. Lee JH, Han IH, Kim DH, et al. Spine computed tomography to magnetic resonance image synthesis using generative adversarial networks: a preliminary study. J Korean Neurosurg Soc 2020 Jan 14 [Epub]. https://doi.org/10.3340/jkns.2019.0084.

47. Bae HJ, Hyun H, Byeon Y, et al. Fully automated 3D segmentation and separation of multiple cervical vertebrae in CT images using a $2 \mathrm{D}$ convolutional neural network. Comput Methods Programs Biomed 2020;184:105119.

48. Jakubicek R, Chmelik J, Jan J, Ourednicek P, Lambert L, Gavelli G. Learning-based vertebra localization and labeling in 3D CT data of possibly incomplete and pathological spines. Comput Methods Programs Biomed 2020;183:105081. 
49. Kim YJ, Ganbold B, Kim KG. Web-based spine segmentation using deep learning in computed tomography images. Healthc Inform Res 2020;26:61-7.

50. Rehman F, Ali Shah SI, Riaz MN, Gilani SO, R F. A region-based deep level set formulation for vertebral bone segmentation of osteoporotic fractures. J Digit Imaging 2020;33:191-203.

51. Dickey JP, Pierrynowski MR, Bednar DA, Yang SX. Relationship between pain and vertebral motion in chronic low-back pain subjects. Clin Biomech (Bristol, Avon) 2002;17:345-52.

52. Lin CS, Chiu JS, Hsieh MH, Mok MS, Li YC, Chiu HW. Predicting hypotensive episodes during spinal anesthesia with the application of artificial neural networks. Comput Methods Programs Biomed 2008;92:193-7.

53. Parsaeian M, Mohammad K, Mahmoudi M, Zeraati $\mathrm{H}$. Comparison of logistic regression and artificial neural network in low back pain prediction: second national health survey. Iran J Public Health 2012;41:86-92.

54. Papic M, Brdar S, Papic V, Loncar-Turukalo T. Return to work after lumbar microdiscectomy: personalizing approach through predictive modeling. Stud Health Technol Inform 2016;224:181-3.

55. Karhade AV, Ogink P, Thio Q, et al. Development of machine learning algorithms for prediction of discharge disposition after elective inpatient surgery for lumbar degenerative disc disorders. Neurosurg Focus 2018;45:E6.

56. Arvind V, Kim JS, Oermann EK, Kaji D, Cho SK. Predicting surgical complications in adult patients undergoing anterior cervical discectomy and fusion using machine learning. Neurospine 2018;15:329-37.

57. Han SS, Azad TD, Suarez PA, Ratliff JK. A machine learning approach for predictive models of adverse events following spine surgery. Spine J 2019;19:177281.

58. DeVries Z, Hoda M, Rivers CS, et al. Development of an unsupervised machine learning algorithm for the prognostication of walking ability in spinal cord injury patients. Spine J 2020;20:213-24.

59. Staartjes VE, de Wispelaere MP, Vandertop WP, Schroder ML. Deep learning-based preoperative predictive analytics for patient-reported outcomes following lumbar discectomy: feasibility of center-specific modeling. Spine J 2019;19:853-61.
60. Karhade AV, Thio QCBS, Ogink PT, et al. Development of machine learning algorithms for prediction of 30-day mortality after surgery for spinal metastasis. Neurosurgery 2019;85:E83-91.

61. Karhade AV, Thio QC, Ogink PT, et al. Predicting 90day and 1-year mortality in spinal metastatic disease: development and internal validation. Neurosurgery 2019;85:E671-81.

62. Weber KA, Smith AC, Wasielewski M, et al. Deep learning convolutional neural networks for the automatic quantification of muscle fat infiltration following whiplash injury. Sci Rep 2019;9:7973.

63. Goyal A, Ngufor C, Kerezoudis P, McCutcheon B, Storlie C, Bydon M. Can machine learning algorithms accurately predict discharge to nonhome facility and early unplanned readmissions following spinal fusion? Analysis of a national surgical registry. J Neurosurg Spine 2019:1-11.

64. Karhade AV, Ogink PT, Thio QCBS, et al. Development of machine learning algorithms for prediction of prolonged opioid prescription after surgery for lumbar disc herniation. Spine J 2019;19:1764-71.

65. Ryu SM, Seo SW, Lee SH. Novel prognostication of patients with spinal and pelvic chondrosarcoma using deep survival neural networks. BMC Med Inform Decis Mak 2020;20:3.

66. Azimi P, Mohammadi HR, Benzel EC, Shahzadi S, Azhari S. Use of artificial neural networks to predict recurrent lumbar disk herniation. J Spinal Disord Tech 2015;28:E161-5.

67. Azimi P, Mohammadi HR, Benzel EC, Shahzadi S, Azhari S. Use of artificial neural networks to decision making in patients with lumbar spinal canal stenosis. J Neurosurg Sci 2017;61:603-11.

68. Karhade AV, Shah AA, Bono CM, et al. Development of machine learning algorithms for prediction of mortality in spinal epidural abscess. Spine J 2019;19:1950-9.

69. Stopa BM, Robertson FC, Karhade AV, et al. Predicting nonroutine discharge after elective spine surgery: external validation of machine learning algorithms. J Neurosurg Spine 2019:1-6.

70. Zhang M, Gong H, Zhang K, Zhang M. Prediction of lumbar vertebral strength of elderly men based on quantitative computed tomography images using machine learning. Osteoporos Int 2019;30:2271-82.

71. Hopkins BS, Yamaguchi JT, Garcia R, et al. Using ma- 
chine learning to predict 30-day readmissions after posterior lumbar fusion: an NSQIP study involving 23,264 patients. J Neurosurg Spine 2019:1-8.

72. Hopkins BS, Mazmudar A, Driscoll C, et al. Using artificial intelligence (AI) to predict postoperative surgical site infection: a retrospective cohort of 4046 posterior spinal fusions. Clin Neurol Neurosurg 2020;192:105718.

73. Mann NH 3rd, Brown MD, Hertz DB, Enger I, Tompkins J. Initial-impression diagnosis using lowback pain patient pain drawings. Spine (Phila $\mathrm{Pa}$ 1976) 1993;18:41-53.

74. Ongphiphadhanakul B, Rajatanavin R, Chailurkit $\mathrm{L}$, et al. Prediction of low bone mineral density in postmenopausal women by artificial neural network model compared to logistic regression model. J Med Assoc Thai 1997;80:508-15.

75. Nussbaum MA, Chaffin DB. Pattern classification reveals intersubject group differences in lumbar muscle recruitment during static loading. Clin Biomech (Bristol, Avon) 1997;12:97-106.

76. Wang L, Buchanan TS. Prediction of joint moments using a neural network model of muscle activations from EMG signals. IEEE Trans Neural Syst Rehabil Eng 2002;10:30-7.

77. Arjmand N, Ekrami O, Shirazi-Adl A, Plamondon A, Parnianpour M. Relative performances of artificial neural network and regression mapping tools in evaluation of spinal loads and muscle forces during static lifting. J Biomech 2013;46:1454-62.

78. Amaritsakul Y, Chao CK, Lin J. Multiobjective optimization design of spinal pedicle screws using neural networks and genetic algorithm: mathematical models and mechanical validation. Comput Math Methods Med 2013;2013:462875.

79. Hu B, Kim C, Ning X, Xu X. Using a deep learning network to recognise low back pain in static standing. Ergonomics 2018;61:1374-81.

80. Aghazadeh F, Arjmand N, Nasrabadi AM. Coupled artificial neural networks to estimate 3D wholebody posture, lumbosacral moments, and spinal loads during load-handling activities. J Biomech 2020;102:109332.

81. Liu Y, Sui X, Liu C, Kuang X, Hu Y. Automatic lumbar spine tracking based on Siamese convolutional network. J Digit Imaging 2020;33:423-30.

82. Khor S, Lavallee D, Cizik AM, et al. Development and validation of a prediction model for pain and functional outcomes after lumbar spine surgery. JAMA Surg 2018;153:634-42.

83. Comparative Effectiveness Research Translation Network. Spine lumbar fusion outcomes calculator [Internet]. Seattle (WA): Comparative Effectiveness Research Translation Network; c2017 [cited 2020 Mar 20]. Available from: https://becertain.shinyapps. io/lumbar_fusion_calculator/.

84. Skeletal Oncology Research Group. Lumbar disc disorder prolonged opioid prescription [Internet]. Boston (MA): Skeletal Oncology Research Group [cited 2020 Mar 20]. Available from: https://sorg-apps.shinyapps.io/lumbardiscopioid/.

85. Skeletal Oncology Research Group. Spinal metastatic disease survival predictions [Internet]. Boston (MA): Skeletal Oncology Research Group [cited 2020 Mar 20]. Available from: https://sorg-apps.shinyapps.io/ spinemetssurvival/.

86. Skeletal Oncology Research Group. Discharge disposition: elective inpatient lumbar degenerative disc disorders [Internet]. Boston (MA): Skeletal Oncology Research Group [cited 2020 Mar 20]. Available from: https://sorg-apps.shinyapps.io/discdisposition/.

87. Skeletal Oncology Research Group. Thirty-day mortality spinal metastatic disease [Internet]. Boston (MA): Skeletal Oncology Research Group [cited 2020 Mar 20]. Available from: https://sorg-apps.shinyapps. io/spinemets/.88. Jiang J, Trundle P, Ren J. Medical image analysis with artificial neural networks. Comput Med Imaging Graph 2010;34:617-31.

89. Jamaludin A, Kadir T, Zisserman A. SpineNet: automated classification and evidence visualization in spinal MRIs. Med Image Anal 2017;41:63-73.

90. Visual Geometry Group, University of Oxford. SpineNet online demo [Internet]. Oxford: Visual Geometry Group, University of Oxford [cited 2020 Mar 20]. Available from: http://zeus.robots.ox.ac.uk/ spinenet/.

91. White AA III, Panjabi MM. Clinical biomechanics of the spine. 2nd ed. Baltimore (MD): Lippincott Williams \& Wilkins; 1990.

92. Barone AV, Helcl J, Sennrich R, Haddow B, Birch A. Deep architectures for neural machine translation [Internet]. Ithaca (NY): arXiv, Cornell University; 2017 [cited 2020 Mar 20]. Available from: https:// arxiv.org/pdf/1707.07631.pdf. 